to appear in the Astronomical Journal

\title{
The Solar Neighborhood XIX: Discovery and Characterization of 33 New Nearby White Dwarf Systems
}

\author{
John P. Subasavage, Todd J. Henry \\ Georgia State University, Atlanta, GA 30302-4106 \\ subasavage@chara.gsu.edu, thenry@chara.gsu.edu \\ P. Bergeron, P. Dufour \\ Département de Physique, Université de Montréal, C.P. 6128, Succ. Centre-Ville, \\ Montréal, Québec H3C 3J\%, Canada \\ bergeron@astro.umontreal.ca, dufourpa@astro.umontreal.ca \\ Nigel C. Hambly \\ Scottish Universities Physics Alliance (SUPA), Institute for Astronomy, University of \\ Edinburgh Royal Observatory, Blackford Hill, Edinburgh EH9 3HJ, Scotland, UK \\ nch@roe.ac.uk \\ Thomas D. Beaulieu \\ Georgia State University, Atlanta, GA 30302-4106 \\ beaulieu@chara.gsu.edu
}

\begin{abstract}
We present spectra for 33 previously unclassified white dwarf systems brighter than $V=17$ primarily in the southern hemisphere. Of these new systems, 26 are DA, 4 are DC, 2 are DZ, and 1 is DQ. We suspect three of these systems are unresolved double degenerates. We obtained VRI photometry for these 33 objects as well as for 23 known white dwarf systems without trigonometric parallaxes, also primarily in the southern hemisphere. For the 56 objects, we converted the photometry values to fluxes and fit them to a spectral energy distribution using the
\end{abstract}


spectroscopy to determine which model to use (i.e. pure hydrogen, pure helium, or metal-rich helium), resulting in estimates of $T_{\text {eff }}$ and distance. Eight of the new and 12 known systems are estimated to be within the NStars and Catalogue of Nearby Stars (CNS) horizons of 25 pc, constituting a potential 18\% increase in the nearby white dwarf sample. Trigonometric parallax determinations are underway via CTIOPI for these 20 systems.

One of the DCs is cool so that it displays absorption in the near infrared. Using the distance determined via trigonometric parallax, we are able to constrain the model-dependent physical parameters and find that this object is most likely a mixed $\mathrm{H} / \mathrm{He}$ atmosphere white dwarf similar to other cool white dwarfs identified in recent years with significant absorption in the infrared due to collision-induced absorptions by molecular hydrogen.

Subject headings: solar neighborhood — white dwarfs — stars: evolution — stars: distances — stars: statistics

\section{Introduction}

The study of white dwarfs (WDs) provides insight to understanding WD formation rates, evolution, and space density. Cool WDs, in particular, provide limits on the age of the Galactic disk and could represent some unknown fraction of the Galactic halo dark matter. Individually, nearby WDs are excellent candidates for astrometric planetary searches because the astrometric signature is greater than for an identical WD system more distant. As a population, a complete volume limited sample is necessary to provide unbiased statistics; however, their intrinsic faintness has allowed some to escape detection.

Of the 18 WDs with trigonometric parallaxes placing them within $10 \mathrm{pc}$ of the Sun (the RECONS sample), all but one have proper motions greater than $1^{\prime \prime} 0 \mathrm{yr}^{-1}$ (94\%). By comparison, of the 230 main sequence systems (as of 01 January 2007) in the RECONS sample, $50 \%$ have proper motions greater than $1.0 \mathrm{yr}^{-1}$. We have begun an effort to reduce this apparent selection bias against slower-moving WDs to complete the census of nearby WDs. This effort includes spectroscopic, photometric, and astrometric initiatives to characterize newly discovered as well as known WDs without trigonometric parallaxes. Utilizing the SuperCOSMOS Sky Survey (SSS) for plate magnitude and proper motion information coupled with data from other recently published proper motion surveys (primarily in the southern hemisphere), we have identified relatively bright WD candidates via reduced proper motion diagrams. 
In this paper, we present spectra for 33 newly discovered WD systems brighter than $V=17.0$. Once an object is spectroscopically confirmed to be a WD (in this paper for the first time or elsewhere in the literature), we obtain CCD photometry to derive $T_{\text {eff }}$ and estimate its distance using a spectral energy distribution (SED) fit and a model atmosphere analysis. If an object's distance estimate is within the NStars (Henry et al. 2003) and CNS (Gliese \& Jahreiß 1991) horizons of 25 pc, it is then added to CTIOPI (Cerro Tololo InterAmerican Observatory Parallax Investigation) to determine its true distance (e.g. Jao et al. 2005, Henry et al. 2006).

\section{Candidate Selection}

We used recent high proper motion (HPM) surveys (Pokorny et al. 2004; Subasavage et al. 2005a, b; Finch et al. 2007) in the southern hemisphere for this work because our long-term astrometric observing program CTIOPI, is based in Chile. To select good WD candidates for spectroscopic observations, plate magnitudes via SSS and 2MASS $J H K_{S}$ are extracted for HPM objects. Each object's $\left(R_{59 \mathrm{~F}}-J\right)$ color and reduced proper motion $(\mathrm{RPM})$ are then plotted. RPM correlates proper motion with proximity, which is certainly not always true; however, it is effective at separating WDs from subdwarfs and main sequence stars. Figure1 displays an RPM diagram for the 33 new WDs presented here. To serve as examples for the locations of subdwarfs and main sequence stars, recent HPM discoveries from the SuperCOSMOS-RECONS (SCR) proper motion survey are also plotted (Subasavage et al. 2005a, b). The solid line represents a somewhat arbitrary cutoff separating subdwarfs and WDs. Targets are selected from the region below the solid line. Note there are four stars below this line that are not represented with asterisks. Three have recently been spectroscopically confirmed as WDs (Subasavage et al., in preparation) and one as a subdwarf (SCR $1227-4541$, denoted by "sd") that fell just below the line at $\left(R_{59 \mathrm{~F}}-J\right)=1.4$ and $H_{R_{59 \mathrm{~F}}}=$ 19.8 (Subasavage et al. 2005b).

Completeness limits $(\mathrm{S} / \mathrm{N}>10)$ for 2 MASS are $J=15.8, H=15.1$, and $K_{S}=14.3$ for uncontaminated point sources (Skrutskie et al. 2006). The use of $J$ provides a more reliable RPM diagram color for objects more than a magnitude fainter than the $K_{S}$ limit, which is particularly important for the WDs (with $\left.\left(J-K_{S}\right)<0.4\right)$ discussed here. Only objects bright enough to have 2MASS magnitudes are included in Figure 1. Consequently, all WD candidates are brighter than $V \sim 17$, and are therefore likely to be nearby. Objects that fall in the WD region of the RPM diagram were cross-referenced with SIMBAD and 
McCook \& Sion (1999)1 to determine those that were previously classified as WDs. The remainder were targeted for spectroscopic confirmation.

The remaining 33 candidates comprise the "new sample" whose spectra are presented in this work, while the "known sample" constitutes the 23 previously identified WD systems without trigonometric parallaxes for which we have complete $V R I J H K_{S}$ data.

\section{Data and Observations}

\subsection{Astrometry and Nomenclature}

The traditional naming convention for WDs uses the object's epoch 1950 equinox 1950 coordinates. Coordinates for the new sample were extracted from 2MASS along with the Julian date of observation. These coordinates were adjusted to account for proper motion from the epoch of 2MASS observation to epoch 2000 (hence epoch 2000 equinox 2000). The coordinates were then transformed to equinox 1950 coordinates using the IRAF procedure precess. Finally, the coordinates were again adjusted (opposite the direction of proper motion) to obtain epoch 1950 equinox 1950 coordinates.

Proper motions were taken from various proper motion surveys in addition to unpublished values obtained via the SCR proper motion survey while recovering previously known HPM objects. Appendix A contains the proper motions used for coordinate sliding as well as J2000 coordinates and alternate names.

\subsection{Spectroscopy}

Spectroscopic observations were taken on five separate observing runs in 2003 October and December, 2004 March and September, and 2006 May at the Cerro Tololo InterAmerican Observatory (CTIO) $1.5 \mathrm{~m}$ telescope as part of the SMARTS Consortium. The Ritchey-Chrétien Spectrograph and Loral 1200×800 CCD detector were used with grating 09, providing $8.6 \AA$ resolution and wavelength coverage from 3500 to $6900 \AA$. Observations consisted of two exposures (typically 20 - 30 minutes each) to permit cosmic ray rejection, followed by a comparison HeAr lamp exposure to calibrate wavelength for each object. Bias subtraction, dome/sky flat-fielding, and extraction of spectra were performed using standard IRAF packages.

\footnotetext{
${ }^{1}$ The current web based catalog can be found at http://heasarc.nasa.gov/W3Browse/all/mcksion.html
} 
A slit width of $2^{\prime \prime}$ was used for the 2003 and 2004 observing runs. Some of these data have flux calibration problems because the slit was not rotated to be aligned along the direction of atmospheric refraction. In conjunction with telescope "jitter", light was sometimes lost preferentially at the red end or the blue end for these data.

A slit width of $6^{\prime \prime}$, used for the 2006 May run, eliminated most of the flux calibration problems even though the slit was not rotated. All observations were taken at an airmass of less than 2.0. Within our wavelength window, the maximum atmospheric differential refraction is less than $3^{\prime \prime}$ (Filippenko 1982). A test was performed to verify that no resolution was lost by taking spectra of a $\mathrm{F}$ dwarf with sharp absorption lines from slit widths of $2^{\prime \prime}$ to $10^{\prime \prime}$ in $2^{\prime \prime}$ increments. Indeed, no resolution was lost.

Spectra for the new DA WDs with $T_{\text {eff }} \geq 10000 \mathrm{~K}$ are plotted in Figure 2 while spectra for the new DA WDs with $T_{\text {eff }}<10000 \mathrm{~K}$ are plotted in Figure 3. Featureless DC spectra are plotted in Figure 4, Spectral plots as well as model fits for unusual objects are described in $\S 4.2$.

\subsection{Photometry}

Optical $V R I$ (Johnson $V$, Kron-Cousins $R I$ ) for the new and known samples was obtained using the CTIO $0.9 \mathrm{~m}$ telescope during several observing runs from 2003 through 2006 as part of the Small and Moderate Aperture Research Telescope System (SMARTS) Consortium. The $2048 \times 2046$ Tektronix CCD camera was used with the Tek 2 VRI filter set2. Standard stars from Graham (1982), Bessel (1990), and Landolt (1992) were observed each night through a range of airmasses to calibrate fluxes to the Johnson-Kron-Cousins system and to calculate extinction corrections.

Bias subtraction and dome flat-fielding (using calibration frames taken at the beginning of each night) were performed using standard IRAF packages. When possible, an aperture $14^{\prime \prime}$ in diameter was used to determine the stellar flux, which is consistent with the aperture used by Landolt (1992) for the standard stars. If cosmic rays fell within this aperture, they were removed before flux extraction. In cases of crowded fields, aperture corrections were applied and ranged from $4^{\prime \prime}$ to $12^{\prime \prime}$ in diameter using the largest aperture possible without including contamination from neighboring sources. Uncertainties in the optical photometry were derived by estimating the internal night-to-night variations as well as the external errors (i.e. fits to the standard stars). A complete discussion of the error analysis can be found in

\footnotetext{
${ }^{2}$ The central wavelengths for $V, R$, and $I$ are 5475,6425 , and $8075 \AA$ respectively.
} 
Henry et al. (2004). We adopt a total error of $\pm 0.03 \mathrm{mag}$ in each band. The final optical magnitudes are listed in Table 1 as well as the number of nights each object was observed.

Infrared $J H K_{S}$ magnitudes and errors were extracted via Aladin from 2MASS and are also listed in Table1. $J H K_{S}$ magnitude errors are, in most cases, significantly larger than for $V R I$, and the errors listed give a measure of the total photometric uncertainty (i.e. include both global and systematic components). In cases when the magnitude error is null, the star is near the magnitude limit of 2MASS and the photometry is not reliable.

\section{Analysis}

\section{1. $\quad$ Modeling of Physical Parameters}

The pure hydrogen, pure helium, and mixed hydrogen and helium model atmospheres used to model the WDs are described at length in Bergeron et al. (2001) and references therein, while the helium-rich models appropriate for DQ and DZ stars are described in Dufour et al. (2005, 2007), respectively. The atmospheric parameters for each star are obtained by converting the optical $V R I$ and infrared $J H K_{S}$ magnitudes into observed fluxes, and by comparing the resulting SEDs with those predicted from our model atmosphere calculations. The first step is accomplished by transforming the magnitudes into average stellar fluxes $f_{\lambda}^{m}$ received at Earth using the calibration of Holberg et al. (2006) for photon counting devices. The observed and model fluxes, which depend on $T_{\text {eff }}, \log g$, and atmospheric composition, are related by the equation

$$
f_{\lambda}^{m}=4 \pi(R / D)^{2} H_{\lambda}^{m}
$$

where $R / D$ is the ratio of the radius of the star to its distance from Earth, and $H_{\lambda}^{m}$ is the Eddington flux, properly averaged over the corresponding filter bandpass. Our fitting

technique relies on the nonlinear least-squares method of Levenberg-Marquardt (Press et al. 1992), which is based on a steepest descent method. The value of $\chi^{2}$ is taken as the sum over all bandpasses of the difference between both sides of eq. (1), weighted by the corresponding photometric uncertainties. We consider only $T_{\text {eff }}$ and the solid angle to be free parameters, and the uncertainties of both parameters are obtained directly from the covariance matrix of the fit. In this study, we simply assume a value of $\log g=8.0$ for each star.

As discussed in Bergeron et al. (1997, 2001), the main atmospheric constituent - hydrogen or helium - is determined by comparing the fits obtained with both compositions, or by the presence of $\mathrm{H} \alpha$ in the optical spectra. For DQ and DZ stars, we rely on the 
procedure outlined in Dufour et al. (2005, 2007), respectively: we obtain a first estimate of the atmospheric parameters by fitting the energy distribution with an assumed value of the metal abundances. We then fit the optical spectrum to measure the metal abundances, and use these values to improve our atmospheric parameters from the energy distribution. This procedure is iterated until a self-consistent photometric and spectroscopic solution is achieved.

The derived values for $T_{\text {eff }}$ for each object are listed in Table 11. Also listed are the spectral types for each object determined based on their spectral features. The DAs have been assigned a half-integer temperature index as defined by McCook \& Sion (1999), where the temperature index equals $50,400 / T_{\text {eff }}$. As an external check, we compare in Figure 5 the photometric effective temperatures for the DA stars in Table 1 with those obtained by fitting the observed Balmer line profiles (Figs. 22 and 3) using the spectroscopic technique developed by Bergeron et al. (1992b), and recently improved by Liebert et al. (2003). Our grid of pure hydrogen, NLTE, and convective model atmospheres is also described in Liebert et al. The uncertainties of the spectroscopic technique are typically of 0.038 dex in $\log g$ and $1.2 \%$ in $T_{\text {eff }}$ according to that study. We adopt a slightly larger uncertainty of $1.5 \%$ in $T_{\text {eff }}$ (Spec) because of the problematic flux calibrations of the pre-2006 data (see $\S 3.2$ ). The agreement shown in Figure 5 is excellent, except perhaps at high temperatures where the photometric determinations become more uncertain. It is possible that the significantly elevated point in Figure 5, WD 0310-624 (labeled), is an unresolved double degenerate (see $\S 4.2)$. We refrain here from using the $\log g$ determinations in our analysis because these are available only for the DA stars in our sample, and also because the spectra are not flux calibrated accurately enough for that purpose.

Once the effective temperature and the atmospheric composition are determined, we calculate the absolute visual magnitude of each star by combining the new calibration of Holberg et al. (2006) with evolutionary models similar to those described in Fontaine et al. (2001) but with $\mathrm{C} / \mathrm{O}$ cores, $q(\mathrm{He}) \equiv \log M_{\mathrm{He}} / M_{\star}=10^{-2}$ and $q(\mathrm{H})=10^{-4}$ (representative of hydrogen-atmosphere WDs), and $q(\mathrm{He})=10^{-2}$ and $q(\mathrm{H})=10^{-10}$ (representative of helium-atmosphere WDs)3. By combining the absolute visual magnitude with the Johnson $V$ magnitude, we derive a first estimate of the distance of each star (reported in Table 1). Errors on the distance estimates incorporate the errors of the photometry values as well as an error of 0.25 dex in $\log g$, which is the measured dispersion of the observed distribution using spectroscopic determinations (see Figure 9 of Bergeron et al. 1992b).

Of the 33 new systems presented here, 5 have distance estimates within 25 pc. Four

${ }^{3}$ see http://www.astro.umontreal.ca/^ bergeron/CoolingModels/ 
more systems require additional attention because distance estimates are derived via other means. Three of these are likely within 25 pc. All four are further discussed in the next section. In total, $20 \mathrm{WD}$ systems ( 8 new and 12 known) are estimated (or determined) to be within $25 \mathrm{pc}$ and one additional common proper motion binary system possibly lies within 25 pc.

\subsection{Comments on Individual Systems}

Here we address unusual and interesting objects.

WD 0121-429 is a DA WD that exhibits Zeeman splitting of $\mathrm{H} \alpha$ and $\mathrm{H} \beta$, thereby making its formal classification DAH. The SED fit to the photometry is superb, yielding a $T_{\text {eff }}$ of $6,369 \pm 137 \mathrm{~K}$. When we compare the strength of the absorption line trio with that predicted using the $T_{\text {eff }}$ from the SED fit, the depth of the absorption appears too shallow. Using the magnetic line fitting procedure outlined in Bergeron et al. (1992a), we must include a $50 \%$ dilution factor to match the observed central line of $\mathrm{H} \alpha$. In light of this, we utilized the trigonometric parallax distance determined via CTIOPI of $17.7 \pm 0.7$ pc (Subasavage et al., in preparation) to further constrain this system. The resulting SED fit, with distance (hence luminosity) as a constraint rather than a variable, implies a mass of $0.43 \pm 0.03 \mathrm{M}_{\odot}$. Given the age of our Galaxy, the lowest mass WD that could have formed is $\sim 0.47 \mathrm{M}_{\odot}$ (Iben \& Renzini 1984). It is extremely unlikely that this WD formed through single star evolution. The most likely scenario is that this is a double degenerate binary with a magnetic DA component and a featureless DC component (necessary to dilute the absorption at $\mathrm{H} \alpha$ ), similar to G62-46 (Bergeron et al. 1993) and LHS 2273 (see Figure 33

of Bergeron et al. 1997). If this interpretation is correct, any number of component masses and luminosities can reproduce the SED fit.

The spectrum and corresponding magnetic fit to the $\mathrm{H} \alpha$ lines (including the dilution) is shown in Figure 6. The viewing angle, $i=65^{\circ}$, is defined as the angle between the dipole axis and the line of sight $(i=0$ corresponds to a pole-on view). The best fit produces a dipole field strength, $B_{d}=9.5 \mathrm{MG}$, and a dipole offset, $a_{z}=0.06$ (in units of stellar radius). The positive value of $a_{z}$ implies that the offset is toward the observer. Only $B_{d}$ is moderately constrained, both $i$ and $a_{z}$ can vary significantly yet still produce a reasonable fit to the data (Bergeron et al. 1992a).

WD 0310-624 is a DA WD that is one of the hottest in the new sample. Because of it's elevation significantly above the equal temperature line (solid) in Figure 5, it is possible that it is an unresolved double degenerate with very different component effective temperatures. 
In fact, this method has been used to identify unresolved double degenerate candidates (i.e. Bergeron et al. 2001).

WD 0511-415 is a DA WD (spectrum is plotted in Figure 2) whose spectral fit produces a $T_{\text {eff }}=10,813 \pm 219 \mathrm{~K}$ and a $\log g=8.21 \pm 0.10$ using the spectral fitting procedure of Liebert et al. (2003). This object lies near the red edge of the ZZ Ceti instability strip as defined by Gianninas et al. (2006). If variable, this object would help to constrain the cool edge of the instability strip in $T_{\text {eff }}, \log g$ parameter space. Follow-up high speed photometry is necessary to confirm variability.

WD 0622-329 is a DAB WD displaying the Balmer lines as well as weaker He I at 4472 and $5876 \AA$. The spectrum, shown in Figure 7, is reproduced best with a model having $T_{\text {eff }} \sim 43,700 \mathrm{~K}$. However, the predicted He II absorption line at $4686 \AA$ for a WD of this $T_{\text {eff }}$ is not present in the spectrum. In contrast, the SED fit to the photometry implies a $T_{\text {eff }}$ of $\sim 10,500 \mathrm{~K}$ (using either pure $\mathrm{H}$ or pure He models). Because the $T_{\text {eff }}$ values are vastly discrepant, we explored the possibility that this spectrum is not characterized by a single temperature. We modeled the spectrum assuming the object was an unresolved double degenerate. The best fit implies one component is a DB with $T_{\text {eff }}=14,170 \pm 1,228 \mathrm{~K}$ and the other component is a DA with $T_{\text {eff }}=9,640 \pm 303 \mathrm{~K}$, similar to the unresolved DA + DB degenerate binary PG $1115+166$ analyzed by Bergeron \& Liebert (2002). One can see from Figure 7 that the spectrum is well modeled under this assumption. We conclude this object is likely a distant (well beyond $25 \mathrm{pc}$ ) unresolved double degenerate.

WD 0840-136 is a DZ WD whose spectrum shows both Ca II ( $\mathrm{H}$ \& K) and Ca I (4226 $\AA$ ) lines as shown in Figure 8. Fits to the photometric data for different atmospheric compositions indicate temperatures of about 4800-5000 K. However, fits to the optical spectrum using the models of Dufour et al. (2007) cannot reproduce simultaneously all three calium lines. This problem is similar to that encountered by Dufour et al. (2007) where the atmospheric parameters for the coolest DZ WDs were considered uncertain because of possible high atmospheric pressure effects. We utilize a photometric relation relevant for WDs of any atmospheric composition, which links $M_{V}$ to $(V-I)$ (Salim et al. 2004) to obtain a distance estimate of $19.3 \pm 3.9$ pc.

WD 1054-226 was observed spectroscopically as part of the Edinburgh-Cape (EC) blue object survey and assigned a spectral type of sdB + (Kilkenny et al. 1997). As is evident in Figure 3, the spectrum of this object is the noisiest of all the spectra presented here and perhaps a bit ambiguous. As an additional check, this object was recently observed using the ESO $3.6 \mathrm{~m}$ telescope and has been confirmed to be a cool DA WD (Bergeron, private communication). 
WD 1105-340 is a DA WD (spectrum is plotted in Figure 2) with a common proper motion companion with separation of $30^{\prime \prime} 6$ at position angle $107.1^{\circ}$. The companion's spectral type is M4Ve with $V_{J}=15.04, R_{\mathrm{KC}}=13.68, I_{\mathrm{KC}}=11.96, J=10.26, H=9.70$, and $K_{S}$ $=9.41$. In addition to the SED derived distance estimate for the $\mathrm{WD}$, we utilize the main sequence distance relations of Henry et al. (2004) to estimate a distance to the red dwarf companion. We obtain a distance estimate of $19.1 \pm 3.0 \mathrm{pc}$ for the companion leaving open the possibility that this system may lie just within 25 pc. A trigonometric parallax determination is currently underway for confirmation.

WD 1149-272 is the only DQ WD discovered in the new sample. This object was observed spectroscopically as part of the Edinburgh-Cape (EC) blue object survey for which no features deeper than $5 \%$ were detected and was labeled a possible DC (Kilkenny et al. 1997). It is identified as having weak $\mathrm{C}_{2}$ swan band absorption at 4737 and $5165 \AA$ and is otherwise featureless. The DQ model reproduces the spectrum reliably and is overplotted in Figure 9. This object is characterized as having $T_{\text {eff }}=6188 \pm 194 \mathrm{~K}$ and a $\log (\mathrm{C} / \mathrm{He})=$ $-7.20 \pm 0.16$.

WD 2008-600 is a DC WD (spectrum is plotted in Figure 4) that is flux deficient in the near infrared, as indicated by the 2MASS magnitudes. The SED fit to the photometry is a poor match to either the pure hydrogen or the pure helium models. A pure hydrogen model provides a slightly better match than a pure helium model, and yields a $T_{\text {eff }}$ of $\sim 3100$ $\mathrm{K}$, thereby placing it in the relatively small sample of ultracool WDs. In order to discern the true nature of this object, we have constrained the model using the distance obtained from the CTIOPI trigonometric parallax of $17.1 \pm 0.4 \mathrm{pc}$ (Subasavage et al., in preparation). This object is then best modeled as having mostly helium with trace amounts of hydrogen (log $(\mathrm{He} / \mathrm{H})=2.61)$ in its atmosphere and has a $T_{\text {eff }}=5078 \pm 221 \mathrm{~K}$ (see Figure 10). A mixed hydrogen and helium composition is required to produce sufficient absorption in the infrared as a result of the collision-induced absorption by molecular hydrogen due to collisions with helium. Such mixed atmospheric compositions have also been invoked to explain the infrared flux deficiency in LHS 1126 (Bergeron et al. 1994) as well as SDSS 1337+00 and LHS 3250 (Bergeron \& Leggett 2002). While WD 2008-600 is likely not an ultracool WD, it is one of the brightest and nearest cool WDs known. Because the 2MASS magnitudes are not very reliable, we intend to obtain additional near-infrared photometry to better constrain the fit.

WD 2138-332 is a DZ WD for which a calcium rich model reproduces the spectrum reliably. The spectrum and the overplotted fit are shown in the bottom panel of Figure 8. Clearly evident in the spectrum are the strong Ca II absorption at 3933 and $3968 \AA$. A weaker $\mathrm{Ca}$ I line is seen at $4226 \AA$. Also seen are Mg I absorption lines at 3829, 3832, and $3838 \AA$ (blended) as well as Mg I at 5167, 5173, and $5184 \AA$ (also blended). Several weak Fe I 
lines from $4000 \AA$ to $4500 \AA$ and again from $5200 \AA$ to $5500 \AA$ are also present. The divergence of the spectrum from the fit toward the red end is likely due to an imperfect flux calibration of the spectrum. This object is characterized as having $T_{\text {eff }}=7188 \pm 291 \mathrm{~K}$ and a $\log$ $(\mathrm{Ca} / \mathrm{He})=-8.64 \pm 0.16$. The metallicity ratios are, at first, assumed to be solar (as defined by Grevesse \& Sauval 1998) and, in this case, the quality of the fit was sufficient without deviation. The corresponding $\log (\mathrm{Mg} / \mathrm{He})=-7.42 \pm 0.16$ and $\log (\mathrm{Fe} / \mathrm{He})=-7.50 \pm 0.16$ for this object.

WD 2157-574 is a DA WD (spectrum is plotted in Figure 3) unique to the new sample in that it displays weak Ca II absorption at 3933 and $3968 \AA$ ( $\mathrm{H}$ and $\mathrm{K}$ ) thereby making its formal classification a DAZ. Possible scenarios that enrich the atmospheres of DAZs include accretion via (1) debris disks, (2) ISM, and (3) cometary impacts (see Kilic et al. 2006 and references therein). The 2 MASS $K_{S}$ magnitude is near the faint limit and is unreliable, but even considering the $J$ and $H$ magnitudes, there appears to be no appreciable near-infrared excess. While this may tentatively rule out the possibility of a debris disk, this object would be an excellent candidate for far-infrared spaced-based studies to ascertain the origin of the enrichment.

\section{Discussion}

WDs represent the end state for stars less massive than $\sim 8 \mathrm{M}_{\odot}$ and are therefore relatively numerous. Because of their intrinsic faintness, only the nearby WD population can be easily characterized and provides the benchmark upon which WD stellar astrophysics is based. It is clear from this work and others (e.g. Holberg et al. 2002; Kawka \& Vennes 2006) that the WD sample is complete, at best, to only 13 pc. Spectroscopic confirmation of new WDs as well as trigonometric parallax determinations for both new and known WDs will lead to a more complete sample and will push the boundary of completeness outward. We estimate that 8 new WDs and an additional 12 known WDs without trigonometric parallaxes are nearer than 25 pc, including one within 10 pc (WD 0141-675). Parallax measurements via CTIOPI are underway for these 20 objects to confirm proximity. This total of 20 WDs within 25 pc constitutes an $18 \%$ increase to the 109 WDs with trigonometric parallaxes $\geq$ 40 mas.

Evaluating the proper motions of the new and known samples within 25 pc indicates that almost double the number of systems have been found with $\mu<1^{\prime \prime}$.0 $\mathrm{yr}^{-1}$ than with $\mu \geq 1^{\prime \prime} .0 \mathrm{yr}^{-1}$ (13 vs 7 , see Table 2). The only WD estimated to be within $10 \mathrm{pc}$ has $\mu>$ $1^{\prime \prime} .0 \mathrm{yr}^{-1}$, although WD $1202-232$ is estimated to be $10.2 \pm 1.7 \mathrm{pc}$ and it's proper motion is small $\left(\mu=0\right.$ ".227 $\left.\mathrm{yr}^{-1}\right)$. 
Because this effort focuses mainly on the southern hemisphere, it is likely that there is a significant fraction of nearby WDs in the northern hemisphere that have also gone undetected. With the recent release of the LSPM-North Catalog (Lépine \& Shara 2005), these objects are identifiable by employing the same techniques used in this work. The challenge is the need for a large scale parallax survey focusing on WDs to confirm proximity. Since the HIPPARCOS mission, only six WD trigonometric parallaxes have been published (Hambly et al. 1999; Smart et al. 2003), and of those, only two are within 25 pc. The USNO parallax program is in the process of publishing trigonometric parallaxes for $\sim 130$ WDs, mostly in the northern hemisphere, although proximity was not a primary motivation for target selection (Dahn, private communication).

In addition to further completing the nearby WD census, the wealth of observational data available from this effort provides reliable constraints on their physical parameters (i.e. $T_{\text {eff }}, \log g$, mass, and radius). Unusual objects are then revealed, such as those discussed in $\S 4.2$. In particular, trigonometric parallaxes help identify WDs that are overluminous, as is the case for WD 0121-429. This object, and others similar to it, are excellent candidates to provide insight into binary evolution. If they can be resolved using high resolution astrometric techniques (i.e. speckle, adaptive optics, or interferometry via Hubble Space Telescope's Fine Guidance Sensors), they may provide astrometric masses, which are fundamental calibrators for stellar structure theory and for the reliability of the theoretical WD mass-radius and initial-to-final-mass relationships. To date, only four WD astrometric masses are known to better than $\sim 5 \%$ (Provencal et al. 1998).

One avenue that is completely unexplored to date is a careful high resolution search for planets around WDs. Theory dictates that the Sun will become a WD, and when it does, the outer planets will remain in orbit (not without transformations of their own, of course). In this scenario, the Sun will have lost more than half of its mass, thereby amplifying the signature induced by the planets. Presumably, this has already occurred in the Milky Way and systems such as these merely await detection. Because of the faintness and spectral signatures of WDs (i.e. few, if any, broad absorption lines), current radial velocity techniques are inadequate for planet detection, leaving astrometric techniques as the only viable option. For a given system, the astrometric signature is inversely related to distance (i.e. the nearer the system, the larger the astrometric signature). This effort aims to provide a complete census of nearby WDs that can be probed for these astrometric signatures using future astrometric efforts. 


\section{Acknowledgments}

The RECONS team at Georgia State University wishes to thank the NSF (grant AST 05-07711), NASA's Space Interferometry Mission, and GSU for their continued support of our study of nearby stars. We also thank the continuing support of the members of the SMARTS consortium, who enable the operations of the small telescopes at CTIO where all of the data in this work were collected. J. P. S. is indebted to Wei-Chun Jao for the use of his photometry reduction pipeline. P. B. is a Cottrell Scholar of Research Corporation and would like to thank the NSERC Canada for its support. N. C. H. would like to thank colleagues in the Wide Field Astronomy Unit at Edinburgh for their efforts contributing to the existence

of the SSS; particular thanks go to Mike Read, Sue Tritton, and Harvey MacGillivray. This work has made use of the SIMBAD, VizieR, and Aladin databases, operated at the CDS in Strasbourg, France. We have also used data products from the Two Micron All Sky Survey, which is a joint project of the University of Massachusetts and the Infrared Processing and Analysis Center, funded by NASA and NSF.

\section{A. Appendix}

In order to ensure correct cross-referencing of names for the new and known WD systems presented here, Table 3 lists additional names found in the literature. Objects for which there is an NLTT designation will also have the corresponding L or LP designations found in the NLTT catalog. This is necessary because the NLTT designations were not published in the

original catalog, but rather are the record numbers in the electronic version of the catalog and have been adopted out of necessity.

\section{REFERENCES}

Bergeron, P., Ruiz, M.-T., \& Leggett, S. K. 1992, ApJ, 400, 315

Bergeron, P., Saffer, R. A., \& Liebert, J. 1992, ApJ, 394, 228

Bergeron, P., Ruiz, M.-T., \& Leggett, S. K. 1993, ApJ, 407, 733

Bergeron, P., Ruiz, M.-T., Leggett, S. K., Saumon, D., \& Wesemael, F. 1994, ApJ, 423, 456

Bergeron, P., Ruiz, M. T., \& Leggett, S. K. 1997, ApJS, 108, 339

Bergeron, P., Leggett, S. K., \& Ruiz, M. T. 2001, ApJS, 133, 413 
Bergeron, P., \& Leggett, S. K. 2002, ApJ, 580, 1070

Bergeron, P., \& Liebert, J. 2002, ApJ, 566, 1091

Bessel, M. S. 1990, A\&AS, 83, 357

Carpenter, J. M. 2001, AJ, 121, 2851

Dufour, P., Bergeron, P., \& Fontaine, G. 2005, ApJ, 627, 404

Dufour, P., Bergeron, P., Liebert, J., Harris, H. C., Knapp, G. R., Anderson, S. F., Hall, P. B., Strauss, M. A., Collinge, M. J., \& Edwards, M. C. 2007, submitted to ApJ

Filippenko, A. V. 1982, PASP, 94, 715

Finch, C. T., Henry, T. J., Subasavage, J. P., Jao, W.-C., Hambly, N. C. 2007, AJ, submitted

Fontaine, G., Brassard, P., \& Bergeron, P. 2001, PASP, 113, 409

Gianninas, A., Bergeron, P., \& Fontaine, G. 2006, AJ, 132, 831

Gliese, W., \& Jahreiß, H. 1991, On: The Astronomical Data Center CD-ROM: Selected Astronomical Catalogs, Vol. I; L.E. Brotzmann, S.E. Gesser (eds.), NASA/Astronomical Data Center, Goddard Space Flight Center, Greenbelt, MD

Graham, J. A. 1982, PASP, 94, 244

Grevesse, N., \& Sauval, A. J. 1998, Space Science Reviews, 85, 161

Hambly, N. C., Smartt, S. J., Hodgkin, S. T., Jameson, R. F., Kemp, S. N., Rolleston, W. R. J., \& Steele, I. A. 1999, MNRAS, 309, L33

Henry, T. J., Walkowicz, L. M., Barto, T. C., \& Golimowski, D. A. 2002, AJ, 123, 2002

Henry, T. J., Backman, D. E., Blackwell, J., Okimura, T., \& Jue, S. 2003, The Future of Small Telescopes In The New Millennium. Volume III - Science in the Shadow of Giants, 111

Henry, T. J., Subasavage, J. P., Brown, M. A., Beaulieu, T. D., Jao, W., \& Hambly, N. C. 2004, AJ, 128, 2460

Henry, T. J., Jao, W.-C., Subasavage, J. P., Beaulieu, T. D., Ianna, P. A., Costa, E., \& Méndez, R. A. 2006, AJ, 132, 2360

Holberg, J. B., Oswalt, T. D., \& Sion, E. M. 2002, ApJ, 571, 512 
Holberg, J. B., \& Bergeron, P. 2006, AJ, 132, 1223

Iben, I., \& Renzini, A. 1984, Phys. Rep., 105, 329

Jao, W.-C., Henry, T. J., Subasavage, J. P., Brown, M. A., Ianna, P. A., Bartlett, J. L., Costa, E., \& Méndez, R. A. 2005, AJ, 129, 1954

Kawka, A., \& Vennes, S. 2006, ApJ, 643, 402

Kilic, M., von Hippel, T., Leggett, S. K., \& Winget, D. E. 2006, ApJ, 646, 474

Kilkenny, D., O’Donoghue, D., Koen, C., Stobie, R. S., \& Chen, A. 1997, MNRAS, 287, 867

Kleinman, S. J., et al. 2004, ApJ, 607, 426

Landolt, A. U. 1992, AJ, 104, 340

Lépine, S., Shara, M. M., \& Rich, R. M. 2003, AJ, 126, 921

Lépine, S., \& Shara, M. M. 2005, AJ, 129, 1483

Lépine, S., Rich, R. M., \& Shara, M. M. 2005, ApJ, 633, L121

Liebert, J., Bergeron, P., \& Holberg, J. B. 2003, AJ, 125, 348

Liebert, J., Bergeron, P., \& Holberg, J. B. 2005, ApJS, 156, 47

Luyten, W. J. 1949, ApJ, 109, 528

Luyten, W. J. 1979, LHS Catalogue (2nd ed.; Minneapolis: Univ. of Minnesota Press)

Luyten, W. J. 1979, New Luyten Catalogue of Stars with Proper Motions Larger than Two Tenths of an Arcsecond (Minneapolis: Univ. of Minnesota Press)

McCook, G. P., \& Sion, E. M. 1999, ApJS, 121, 1

Oppenheimer, B. R., Hambly, N. C., Digby, A. P., Hodgkin, S. T., \& Saumon, D. 2001, Science, 292, 698

Press, W. H., Teukolsky, S. A., Vetterling, W. T., \& Flannery, B. P. 1992, Numerical Recipes in FORTRAN, 2nd edition (Cambridge: Cambridge University Press), 644

Provencal, J. L., Shipman, H. L., Hog, E., \& Thejll, P. 1998, ApJ, 494, 759

Pokorny, R. S., Jones, H. R. A., Hambly, N. C., \& Pinfield, D. J. 2004, A\&A, 421, 763 
Salim, S., Rich, R. M., Hansen, B. M., Koopmans, L. V. E., Oppenheimer, B. R., \& Blandford, R. D. 2004, ApJ, 601, 1075

Scholz, R.-D., Szokoly, G. P., Andersen, M., Ibata, R., \& Irwin, M. J. 2002, ApJ, 565, 539

Skrutskie, M. F., et al. 2006, AJ, 131, 1163

Smart, R. L., et al. 2003, A\&A, 404, 317

Subasavage, J. P., Henry, T. J., Hambly, N. C., Brown, M. A., \& Jao, W. 2005, AJ, 129, 413

Subasavage, J. P., Henry, T. J., Hambly, N. C., Brown, M. A., Jao, W.-C., \& Finch, C. T. 2005, AJ, 130, 1658 
Table 1. Optical and Infrared Photometry, and Derived Parameters for New and Known White Dwarfs.

\begin{tabular}{|c|c|c|c|c|c|c|c|c|c|c|c|c|c|c|c|}
\hline $\begin{array}{l}\text { WD } \\
\text { Name }\end{array}$ & $V_{J}$ & $R_{C}$ & $I_{C}$ & $\begin{array}{c}\# \\
\text { Obs }\end{array}$ & $J$ & $\sigma_{J}$ & $H$ & $\sigma_{H}$ & $K_{S}$ & $\sigma_{K_{S}}$ & $\begin{array}{l}T_{\text {eff }} \\
(\mathrm{K})\end{array}$ & Comp & $\begin{array}{l}\text { Dist } \\
\text { (pc) }\end{array}$ & $\mathrm{SpT}$ & Notes \\
\hline \multicolumn{16}{|c|}{ New Spectroscopically Confirmed White Dwarfs } \\
\hline $0034-602 \ldots$ & 14.08 & 14.19 & 14.20 & 3 & 14.37 & 0.04 & 14.55 & 0.06 & 14.52 & 0.09 & $14655 \pm 1413$ & $\mathrm{H}$ & $35.8 \pm 5.7$ & DA3.5 & \\
\hline $0121-429 \ldots \ldots$ & 14.83 & 14.52 & 14.19 & 4 & 13.85 & 0.02 & 13.63 & 0.04 & 13.53 & 0.04 & $6369 \pm 137$ & $\mathrm{H}$ & $\cdots \pm \cdots$ & $\mathrm{DAH}$ & a \\
\hline $0216-398 \ldots \ldots \ldots \ldots \ldots$ & 15.75 & 15.55 & 15.29 & 3 & 15.09 & 0.04 & 14.83 & 0.06 & 14.89 & 0.14 & $7364 \pm 241$ & $\mathrm{H}$ & $29.9 \pm 4.7$ & DA7.0 & \\
\hline $0253-755 \ldots \ldots \ldots \ldots . . . .$. & 16.70 & 16.39 & 16.08 & 2 & 15.77 & 0.07 & 15.76 & 0.15 & 15.34 & null & $6235 \pm 253$ & $\mathrm{He}$ & $34.7 \pm 5.5$ & $\mathrm{DC}$ & \\
\hline $0310-624 \ldots \ldots \ldots \ldots \ldots$ & 15.92 & 15.99 & 16.03 & 2 & 16.13 & 0.10 & 16.31 & 0.27 & 16.50 & null & $13906 \pm 1876$ & $\mathrm{H}$ & $\cdots \pm \cdots$ & DA3.5 & b \\
\hline $0344+014 \ldots \ldots \ldots \ldots$ & 16.52 & 16.00 & 15.54 & 2 & 15.00 & 0.04 & 14.87 & 0.09 & 14.70 & 0.12 & $5084 \pm \quad 91$ & $\mathrm{He}$ & $19.9 \pm 3.1$ & $\mathrm{DC}$ & \\
\hline $0404-510 \ldots \ldots \ldots \ldots \ldots$ & 15.81 & 15.76 & 15.70 & 2 & 15.74 & 0.06 & 15.55 & 0.13 & 15.59 & null & $10052 \pm 461$ & $\mathrm{H}$ & $53.5 \pm 8.5$ & DA5.0 & \\
\hline $0501-555 \ldots \ldots \ldots \ldots \ldots$ & 16.35 & 16.17 & 15.98 & 2 & 15.91 & 0.08 & 15.72 & 0.15 & 15.82 & 0.26 & $7851 \pm 452$ & $\mathrm{He}$ & $44.8 \pm 6.9$ & $\mathrm{DC}$ & \\
\hline $0511-415 \ldots \ldots \ldots \ldots . . .$. & 16.00 & 15.99 & 15.93 & 2 & 15.96 & 0.08 & 15.97 & 0.15 & 15.20 & null & $10393 \pm 560$ & $\mathrm{H}$ & $61.8 \pm 10.8$ & DA5.0 & \\
\hline $0525-311 \ldots \ldots \ldots \ldots \ldots$ & 15.94 & 16.03 & 16.03 & 2 & 16.20 & 0.12 & 16.21 & 0.25 & 14.98 & null & $12941 \pm 1505$ & $\mathrm{H}$ & $76.3 \pm 13.6$ & DA4.0 & \\
\hline $0607-530 \ldots \ldots \ldots \ldots . . .$. & 15.99 & 15.92 & 15.78 & 3 & 15.82 & 0.07 & 15.66 & 0.14 & 15.56 & 0.21 & $9395 \pm 426$ & $\mathrm{H}$ & $51.7 \pm 9.0$ & DA5.5 & \\
\hline $0622-329 \ldots \ldots$ & 15.47 & 15.41 & 15.36 & 2 & 15.44 & 0.06 & 15.35 & 0.11 & 15.53 & 0.25 & $\cdots \pm \cdots$ & $\ldots$ & $\cdots \pm \cdots$ & DAB & c \\
\hline $0821-669 \ldots$ & 15.34 & 14.82 & 14.32 & 3 & 13.79 & 0.03 & 13.57 & 0.03 & 13.34 & 0.04 & $5160 \pm \quad 95$ & $\mathrm{H}$ & $11.5 \pm 1.9$ & DA10.0 & \\
\hline $0840-136 \ldots$ & 15.72 & 15.36 & 15.02 & 3 & 14.62 & 0.03 & 14.42 & 0.05 & 14.54 & 0.09 & $\cdots \pm \cdots$ & $\ldots$ & $\cdots \pm \cdots$ & $\mathrm{DZ}$ & d \\
\hline $1016-308 \ldots$ & 14.67 & 14.75 & 14.81 & 2 & 15.05 & 0.04 & 15.12 & 0.08 & 15.41 & 0.21 & $16167 \pm 1598$ & $\mathrm{H}$ & $50.6 \pm 9.2$ & DA3.0 & \\
\hline $1054-226 \ldots$ & 16.02 & 15.82 & 15.62 & 2 & 15.52 & 0.05 & 15.40 & 0.11 & 15.94 & 0.26 & $8266 \pm 324$ & $\mathrm{H}$ & $41.0 \pm 7.0$ & DA6.0 & e \\
\hline $1105-340 \ldots \ldots$ & 13.66 & 13.72 & 13.79 & 2 & 13.95 & 0.03 & 13.98 & 0.04 & 14.05 & 0.07 & $13926 \pm 988$ & $\mathrm{H}$ & $28.2 \pm 4.8$ & DA 3.5 & $\mathrm{f}$ \\
\hline $1149-272 \ldots \ldots$ & 15.87 & 15.59 & 15.37 & 4 & 15.17 & 0.05 & 14.92 & 0.06 & 14.77 & 0.11 & $6188 \pm 194$ & $\mathrm{He}(+\mathrm{C})$ & $24.0 \pm 3.8$ & $\mathrm{DQ}$ & \\
\hline $1243-123 \ldots$ & 15.57 & 15.61 & 15.64 & 2 & 15.74 & 0.07 & 15.73 & 0.11 & 16.13 & null & $12608 \pm 1267$ & $\mathrm{H}$ & $62.6 \pm 10.7$ & DA4.0 & \\
\hline $1316-215 \ldots \ldots \ldots \ldots \ldots$ & 16.67 & 16.33 & 15.99 & 2 & 15.56 & 0.05 & 15.33 & 0.08 & 15.09 & 0.14 & $6083 \pm 201$ & $\mathrm{H}$ & $31.6 \pm 5.3$ & DA 8.5 & \\
\hline $1436-781 \ldots \ldots \ldots \ldots \ldots$ & 16.11 & 15.82 & 15.49 & 2 & 15.04 & 0.04 & 14.88 & 0.08 & 14.76 & 0.14 & $6246 \pm 200$ & $\mathrm{H}$ & $26.0 \pm 4.3$ & DA8.0 & \\
\hline $1452-310 \ldots \ldots \ldots \ldots . . . . .$. & 15.85 & 15.77 & 15.63 & 2 & 15.58 & 0.06 & 15.54 & 0.09 & 15.50 & 0.22 & $9206 \pm 375$ & $\mathrm{H}$ & $46.8 \pm 8.1$ & DA 5.5 & \\
\hline $1647-327 \ldots \ldots \ldots \ldots \ldots$ & 16.21 & 15.85 & 15.49 & 3 & 15.15 & 0.05 & 14.82 & 0.08 & 14.76 & 0.11 & $6092 \pm 193$ & $\mathrm{H}$ & $25.5 \pm 4.2$ & DA 8.5 & \\
\hline $1742-722 \ldots \ldots \ldots \ldots$ & 15.53 & 15.62 & 15.70 & 2 & 15.85 & 0.08 & 15.99 & 0.18 & 15.65 & null & $15102 \pm 2451$ & $\mathrm{H}$ & $71.7 \pm 12.9$ & DA3.5 & \\
\hline $1946-273 \ldots \ldots$ & 14.19 & 14.31 & 14.47 & 2 & 14.72 & 0.04 & 14.77 & 0.09 & 14.90 & 0.13 & $21788 \pm 3304$ & $\mathrm{H}$ & $52.0 \pm 9.9$ & DA2.5 & \\
\hline $2008-600 \ldots$ & 15.84 & 15.40 & 14.99 & 4 & 14.93 & 0.05 & 15.23 & 0.11 & 15.41 & null & $5078 \pm 221$ & $\mathrm{He}$ & $\cdots \pm \cdots$ & $\mathrm{DC}$ & $\mathrm{g}$ \\
\hline $2008-799 \ldots \ldots \ldots \ldots$ & 16.35 & 15.96 & 15.57 & 3 & 15.11 & 0.04 & 15.03 & 0.08 & 14.64 & 0.09 & $5807 \pm 161$ & $\mathrm{H}$ & $24.5 \pm 4.1$ & DA 8.5 & \\
\hline $2035-369 \ldots \ldots \ldots \ldots$ & 14.94 & 14.85 & 14.72 & 2 & 14.75 & 0.04 & 14.72 & 0.06 & 14.84 & 0.09 & $9640 \pm 298$ & $\mathrm{H}$ & $33.1 \pm 5.7$ & DA 5.0 & \\
\hline $2103-397 \ldots \ldots \ldots \ldots \ldots$ & 15.31 & 15.15 & 14.91 & 2 & 14.79 & 0.03 & 14.63 & 0.04 & 14.64 & 0.08 & $7986 \pm 210$ & $\mathrm{H}$ & $28.2 \pm 4.8$ & DA 6.5 & \\
\hline $2138-332 \ldots \ldots \ldots \ldots \ldots$ & 14.47 & 14.30 & 14.16 & 3 & 14.17 & 0.03 & 14.08 & 0.04 & 13.95 & 0.06 & $7188 \pm 291$ & $\mathrm{He}(+\mathrm{Ca})$ & $17.3 \pm 2.7$ & $\mathrm{DZ}$ & \\
\hline $2157-574 \ldots \ldots \ldots \ldots$ & 15.96 & 15.73 & 15.49 & 3 & 15.18 & 0.04 & 15.05 & 0.07 & 15.28 & 0.17 & $7220 \pm 246$ & $\mathrm{H}$ & $32.0 \pm 5.4$ & DAZ & \\
\hline $2218-416 \ldots \ldots \ldots \ldots$ & 15.36 & 15.35 & 15.24 & 2 & 15.38 & 0.04 & 15.14 & 0.09 & 15.39 & 0.15 & $10357 \pm 414$ & $\mathrm{H}$ & $45.6 \pm 8.0$ & DA5.0 & \\
\hline $2231-387 \ldots$ & 16.02 & 15.88 & 15.62 & 2 & 15.57 & 0.06 & 15.51 & 0.11 & 15.11 & 0.15 & $8155 \pm 336$ & $\mathrm{H}$ & $40.6 \pm 6.9$ & DA6.0 & \\
\hline \multicolumn{16}{|c|}{ Known White Dwarfs without a Trigonometric Parallax Estimated to be Within $25 \mathrm{pc}$} \\
\hline $0141-675 \ldots \ldots \ldots \ldots$ & 13.82 & 13.52 & 13.23 & 3 & 12.87 & 0.02 & 12.66 & 0.03 & 12.58 & 0.03 & $6484 \pm 128$ & $\mathrm{H}$ & $9.7 \pm 1.6$ & DA8.0 & \\
\hline $0806-661 \ldots \ldots \ldots \ldots$ & 13.73 & 13.66 & 13.61 & 3 & 13.70 & 0.02 & 13.74 & 0.03 & 13.78 & 0.04 & $10753 \pm 406$ & $\mathrm{He}$ & $21.1 \pm 3.5$ & $\mathrm{DQ}$ & \\
\hline $1009-184 \ldots \ldots \ldots \ldots . . . .$. & 15.44 & 15.18 & 14.91 & 3 & 14.68 & 0.04 & 14.52 & 0.05 & 14.31 & 0.07 & $6449 \pm 194$ & $\mathrm{He}$ & $20.9 \pm 3.2$ & DZ & $\mathrm{h}$ \\
\hline $1036-204 \ldots \ldots \ldots \ldots . . .$. & 16.24 & 15.54 & 15.34 & 3 & 14.63 & 0.03 & 14.35 & 0.04 & 14.03 & 0.07 & $4948 \pm \quad 70$ & $\mathrm{He}$ & $16.2 \pm 2.5$ & $\mathrm{DQ}$ & i \\
\hline $1202-232$ & 12.80 & 12.66 & 12.52 & 3 & 12.40 & 0.02 & 12.30 & 0.03 & 12.34 & 0.03 & $8623 \pm 168$ & $\mathrm{H}$ & $10.2 \pm 1.7$ & DA6.0 & \\
\hline $1315-781$. & 16.16 & 15.73 & 15.35 & 2 & 14.89 & 0.04 & 14.67 & 0.08 & 14.58 & 0.12 & $5720 \pm 162$ & $\mathrm{H}$ & $21.6 \pm 3.6$ & $\mathrm{DC}$ & j \\
\hline $1339-340$ & 16.43 & 16.00 & 15.56 & 2 & 15.00 & 0.04 & 14.75 & 0.06 & 14.65 & 0.10 & $5361 \pm 138$ & $\mathrm{H}$ & $21.2 \pm 3.5$ & DA9.5 & \\
\hline $1756+143$. & 16.30 & 16.12 & 15.69 & 1 & 14.93 & 0.04 & 14.66 & 0.06 & 14.66 & 0.08 & $5466 \pm 151$ & $\mathrm{H}$ & $22.4 \pm 3.4$ & DA9.0 & $\mathrm{k}$ \\
\hline
\end{tabular}


Table 1-Continued

\begin{tabular}{|c|c|c|c|c|c|c|c|c|c|c|c|c|c|c|c|}
\hline $\begin{array}{c}\text { WD } \\
\text { Name }\end{array}$ & $V_{J}$ & $R_{C}$ & $I_{C}$ & $\begin{array}{c}\# \\
\text { Obs }\end{array}$ & $J$ & $\sigma_{J}$ & $H$ & $\sigma_{H}$ & $K_{S}$ & $\sigma_{K_{S}}$ & $\begin{array}{c}T_{\text {eff }} \\
(\mathrm{K}) \\
\end{array}$ & Comp & $\begin{array}{l}\text { Dist } \\
\text { (pc) }\end{array}$ & $\mathrm{SpT}$ & Notes \\
\hline $1814+134$. & 15.85 & 15.34 & 14.86 & 2 & 14.38 & 0.04 & 14.10 & 0.06 & 14.07 & 0.06 & $5313 \pm 115$ & $\mathrm{H}$ & $15.6 \pm 2.5$ & DA9.5 & \\
\hline $2040-392 \ldots \ldots \ldots \ldots$ & 13.74 & 13.77 & 13.68 & 2 & 13.77 & 0.02 & 13.82 & 0.03 & 13.81 & 0.05 & $10811 \pm 325$ & $\mathrm{H}$ & $23.1 \pm 4.0$ & DA 4.5 & \\
\hline $2226-754 \mathrm{~B} \ldots$ & 16.88 & 16.17 & 15.51 & 2 & 14.86 & 0.04 & 14.82 & 0.06 & 14.72 & 0.12 & $4177 \pm 112$ & $\mathrm{H}$ & $14.0 \pm 2.2$ & DC & 1 \\
\hline \multicolumn{16}{|c|}{ Known White Dwarfs without a Trigonometric Parallax Estimated to be Beyond 25 pc } \\
\hline $0024-556 \ldots \ldots \ldots \ldots$ & 15.17 & 15.15 & 15.07 & 2 & 15.01 & 0.04 & 15.23 & 0.10 & 15.09 & 0.14 & $10007 \pm 378$ & $\mathrm{H}$ & $39.8 \pm 6.8$ & DA 5.0 & \\
\hline $0442-304 \ldots \ldots \ldots \ldots$ & 16.03 & 15.93 & 15.86 & 2 & 15.94 & 0.09 & 15.81 & null & 15.21 & null & $9949 \pm 782$ & $\mathrm{He}$ & $55.1 \pm 9.1$ & DQ & \\
\hline $0928-713 \ldots \ldots \ldots \ldots$ & 15.11 & 14.97 & 14.83 & 3 & 14.77 & 0.03 & 14.69 & 0.06 & 14.68 & 0.09 & $8836 \pm 255$ & $\mathrm{H}$ & $30.7 \pm 5.3$ & DA 5.5 & \\
\hline $1143-013 \ldots \ldots \ldots \ldots \ldots$ & 16.39 & 16.08 & 15.79 & 1 & 15.54 & 0.06 & 15.38 & 0.08 & 15.18 & 0.16 & $6824 \pm 250$ & $\mathrm{H}$ & $34.4 \pm 5.8$ & DA 7.5 & \\
\hline $1237-230 \ldots \ldots \ldots \ldots$ & 16.53 & 16.13 & 15.74 & 2 & 15.35 & 0.05 & 15.08 & 0.08 & 14.94 & 0.11 & $5841 \pm 173$ & $\mathrm{H}$ & $26.9 \pm 4.5$ & DA8.5 & \\
\hline $1314-153 \ldots \ldots \ldots \ldots \ldots$ & 14.82 & 14.89 & 14.97 & 2 & 15.17 & 0.05 & 15.26 & 0.09 & 15.32 & 0.21 & $15604 \pm 2225$ & $\mathrm{H}$ & $52.7 \pm 9.5$ & DA3.0 & \\
\hline $1418-088 \ldots \ldots \ldots \ldots . . .$. & 15.39 & 15.21 & 15.01 & 2 & 14.76 & 0.04 & 14.73 & 0.06 & 14.76 & 0.10 & $7872 \pm 243$ & $\mathrm{H}$ & $28.5 \pm 4.8$ & DA 6.5 & \\
\hline $1447-190 \ldots \ldots \ldots \ldots$ & 15.80 & 15.59 & 15.32 & 2 & 15.06 & 0.04 & 14.87 & 0.07 & 14.78 & 0.11 & $7153 \pm 235$ & $\mathrm{H}$ & $29.1 \pm 4.9$ & DA 7.0 & \\
\hline $1607-250 \ldots \ldots \ldots \ldots$ & 15.19 & 15.12 & 15.09 & 2 & 15.08 & 0.08 & 15.08 & 0.08 & 15.22 & 0.15 & $10241 \pm 457$ & $\mathrm{H}$ & $41.2 \pm 7.2$ & DA5.0 & \\
\hline
\end{tabular}

${ }^{a}$ Distance via SED fit (not listed) is underestimated because object is likely an unresolved double degenerate with one magnetic component (see $\S 4.2$ ). Instead, we adopt the trigonometric parallax distance of $17.7 \pm 0.7 \mathrm{pc}$ derived via CTIOPI.

${ }^{b}$ Distance via SED fit (not listed) is underestimated because object is likely a distant (well beyond $25 \mathrm{pc}$ ) unresolved double degenerate (see $\S 4.2$ ).

${ }^{c}$ Distance via SED fit (not listed) is underestimated because object is likely a distant (well beyond $25 \mathrm{pc}$ ) unresolved double degenerate with components of type DA and DB (see § 4.2). Temperatures derived from the spectroscopic fit yield 9,640 $\pm 303 \mathrm{~K}$ and 14,170 $\pm 1,228 \mathrm{~K}$ for the DA and DB respectively.

${ }^{\mathrm{d}}$ Object is likely cooler than $T_{\text {eff }} \sim 5000 \mathrm{~K}$ and the theoretical models do not provide an accurate treatment at these temperatures (see § 4.2). Instead, we use the linear photometric distance relation of Salim et al. (2004) and obtain a distance estimate of $19.3 \pm 3.9 \mathrm{pc}$.

${ }^{\mathrm{e}}$ This object was observed as part of the Edinburgh-Cape survey and was classified as a sdB+ (Kilkenny et al. 1997).

${ }^{\mathrm{f}}$ Distance of $19.1 \pm 3.0 \mathrm{pc}$ is estimated using VRIJHKS for the common proper motion companion M dwarf and the relations of Henry et al. (2004). System is possibly within 25 pc. (see $\S 4.2$ ).

${ }^{\mathrm{g}}$ Distance estimate is undetermined. Instead, we adopt the distance measured via trogonometric parallax of $17.1 \pm 0.4 \mathrm{pc}$ (see $\S 4.2$ ).

${ }^{\mathrm{h}}$ Not listed in McCook \& Sion (1999) but identified as a DC/DQ WD by Henry et al. (2002). We obtained blue spectra that show Ca II H \& K absorption and classify this object as a DZ.

${ }^{i}$ The SED fit to the photometry is marginal. This object displays deep swan band absorption that significantly affects its measured magnitudes.

${ }^{j}$ Not listed in McCook \& Sion (1999) but identified as a WD by Luvten (1949). Spectral type is derived from our spectra.

${ }^{k}$ As of mid-2004, object has moved onto a background source. Photometry is probably contaminated, which is consistent with the poor SED fit for this object.

${ }^{1}$ Spectral type was determined using spectra published by Scholz et al. (2002). 
Table 2. Distance Estimate Statistics for New and Known White Dwarfs.

\begin{tabular}{|c|c|c|c|}
\hline Proper motion & $\mathrm{d} \leq 10 \mathrm{pc}$ & $10 \mathrm{pc}<\mathrm{d} \leq 25 \mathrm{pc}$ & $\mathrm{d}>25 \mathrm{pc}$ \\
\hline$\mu \geq 1^{\prime \prime} 0 \mathrm{yr}^{-1} \ldots \ldots \ldots \ldots \ldots \ldots$ & 1 & 6 & 1 \\
\hline $1^{\prime \prime} 0 \mathrm{yr}^{-1}>\mu \geq 00^{\prime \prime} 8 \mathrm{yr}^{-1} \ldots \ldots$ & 0 & 0 & 0 \\
\hline $0^{\prime \prime} 8 \mathrm{yr}^{-1}>\mu \geq 00^{\prime \prime} 6 \mathrm{yr}^{-1} \ldots \ldots$ & 0 & 2 & 2 \\
\hline $0^{\prime \prime} 6 \mathrm{yr}^{-1}>\mu \geq 00^{\prime \prime} 4 \mathrm{yr}^{-1} \ldots \ldots$ & 0 & 6 & 11 \\
\hline $0^{\prime \prime} 4 \mathrm{yr}^{-1}>\mu \geq 00^{\prime \prime} 18 \mathrm{yr}^{-1} \ldots$ & 0 & 5 & 22 \\
\hline 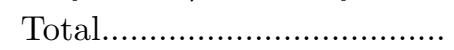 & 1 & 19 & 36 \\
\hline
\end{tabular}


Table 3. Astrometry and Alternate Designations for New and Known White Dwarfs.

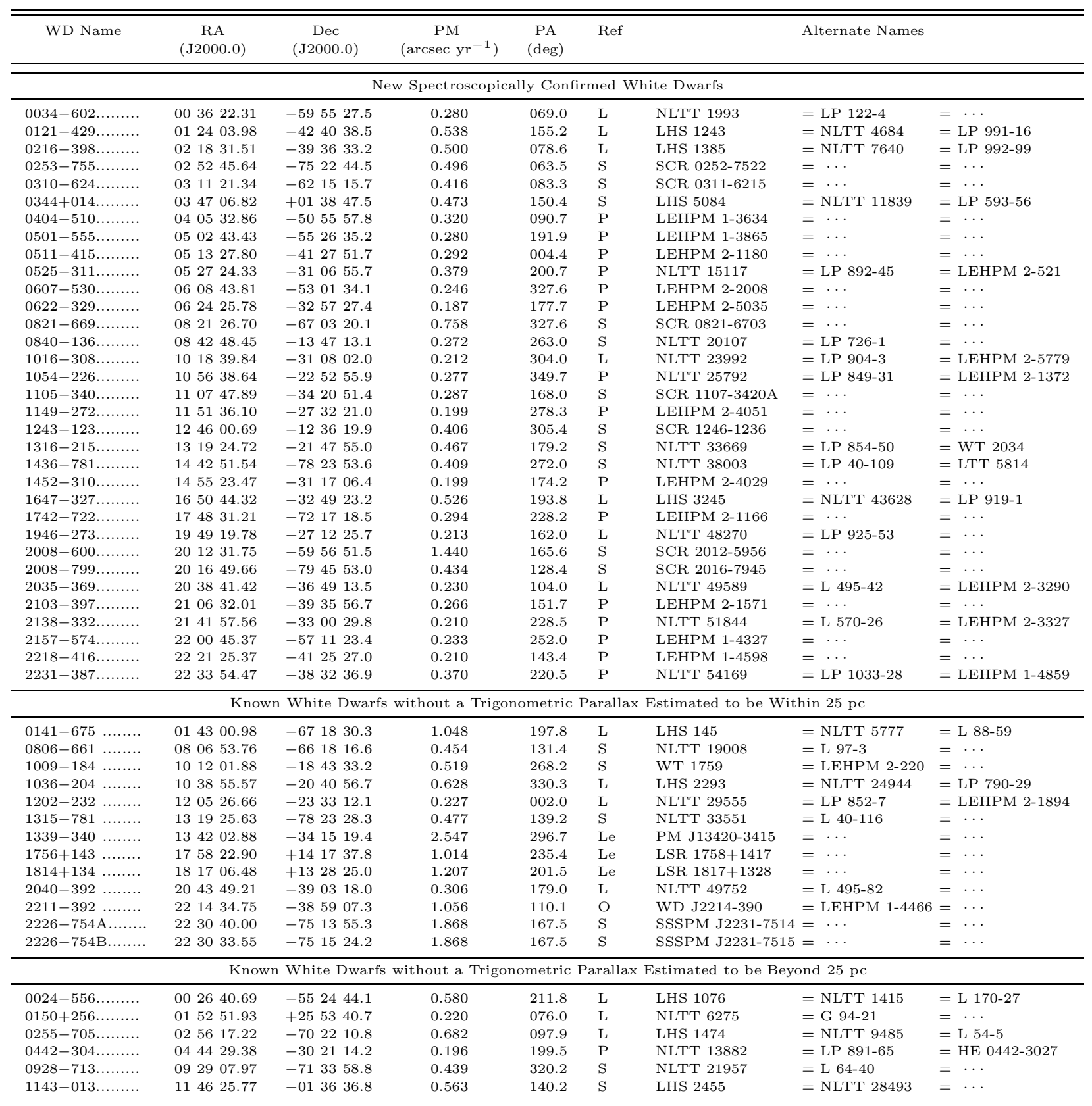


Table 3-Continued

\begin{tabular}{|c|c|c|c|c|c|c|c|}
\hline WD Name & $\begin{array}{c}\text { RA } \\
(\mathrm{J} 2000.0)\end{array}$ & $\begin{array}{c}\text { Dec } \\
(\mathrm{J} 2000.0)\end{array}$ & $\begin{array}{c}\mathrm{PM} \\
\left(\operatorname{arcsec} \mathrm{yr}^{-1}\right)\end{array}$ & $\begin{array}{l}\text { PA } \\
(\mathrm{deg})\end{array}$ & Ref & & Alternate Names \\
\hline $1237-230 \ldots \ldots \ldots$ & 124024.18 & -231743.8 & 1.102 & 219.9 & $\mathrm{~L}$ & LHS 339 & $=$ NLTT $31473=$ LP 853-15 \\
\hline $1314-153 \ldots \ldots \ldots$ & 131643.59 & -153558.3 & 0.708 & 196.7 & $\mathrm{~L}$ & LHS 2712 & $=$ NLTT $33503=$ LP 737-47 \\
\hline $1418-088 \ldots \ldots \ldots$ & 142054.93 & -090508.7 & 0.480 & 266.8 & S & LHS 5270 & $=\mathrm{NLTT} 37026=\cdots$ \\
\hline $1447-190 \ldots \ldots \ldots$ & 145011.93 & -191408.7 & 0.253 & 285.4 & $\mathrm{P}$ & NLTT 38499 & $=\mathrm{LP} 801-14=$ LEHPM $2-1835$ \\
\hline $1607-250 \ldots \ldots \ldots$ & $16 \quad 10 \quad 50.21$ & $-2513 \quad 16.0$ & 0.209 & 314.0 & $\mathrm{~L}$ & NLTT 42153 & $=\mathrm{LP} 861-31$ \\
\hline
\end{tabular}

References. - (L) Luvten 1979a b, (Le) Lépine et al. 2003, Lépine et al. 2005, (O) Oppenheimer et al. 2001, (P) Pokornv et al. 2004, (S) Subasavage et al. 2005a b, this work 
Fig. 1.- Reduced proper motion diagram used to select WD candidates for spectroscopic follow-up. Plotted are the new high proper motion objects from Subasavage et al. (2005a,b). The line is a somewhat arbitrary boundary between the WDs (below) and the subdwarfs (just above). Main sequence dwarfs fall above and to the right of the subdwarfs, although there is significant overlap. Asterisks indicate the 33 new WDs reported here. Three dots in the WD region are deferred to a future paper. The point labeled "sd" is a confirmed subdwarf contaminant of the WD sample.

Fig. 2.- Spectral plots of the hot $\left(T_{\text {eff }} \geq 10000 \mathrm{~K}\right)$ DA WDs from the new sample, plotted in descending $T_{\text {eff }}$ as derived from the SED fits to the photometry. Note that some of the flux calibrations are not perfect, in particular, at the blue end.

Fig. 3.- Spectral plots of cool $\left(T_{\text {eff }}<10000 \mathrm{~K}\right)$ DA WDs from the new sample, plotted in descending $T_{\text {eff }}$ as derived from the SED fits to the photometry. Note that some of the flux calibrations are not perfect, in particular, at the blue end.

Fig. 4.- Spectral plots of the four featureless DC white dwarfs from the new sample, plotted in descending $T_{\text {eff }}$ as derived from the SED fits to the photometry. Note that some of the flux calibrations are not perfect, in particular, at the blue end.

Fig. 5.- Comparison plot of the values of $T_{\text {eff }}$ derived from photometric SED fitting vs those derived from spectral fitting for 25 of the DA WDs in the new sample. The solid line represents equal temperatures. The elevated point, 0310-624, is discussed in $\S 4.2$.

Fig. 6.- Spectral plot of WD 0121-429. The inset plot displays the spectrum (light line) in the $\mathrm{H} \alpha$ region to which a magnetic fit (heavy line), as outlined in Bergeron et al. (1992a), was performed using the $T_{\text {eff }}$ obtained from the SED fit to the photometry. The resulting magnetic parameters are listed below the fit.

Fig. 7.- Spectral plot of WD 0622-329. The inset plot displays the spectrum (light line) in the region to which the model (heavy line) was fit assuming the spectrum is a convolution of a DB component and a slightly cooler DA component. Best fit physical parameters are listed below the fit for each component.

Fig. 8.- (top panel) Spectral plot of WD 0840-136. The DZ model failed to reproduce the spectrum presumably because this object is cooler than $T_{\text {eff }} \sim 5000 \mathrm{~K}$ where additional pressure effects, not included in the model, become important. (bottom panel) Spectral plot of WD 2138-332. The inset plot displays the spectrum (light line) in the region to which the model (heavy line) was fit. 
Fig. 9.- Spectral plot of WD 1149-272. The inset plot displays the spectrum (light line) in the region to which the model (heavy line) was fit.

Fig. 10.- Spectral energy distribution plot of WD 2008-600 with the distance constrained by the trigonometric distance of $17.1 \pm 0.4$ pc. Best fit physical parameters are listed below the fit. Points are fit values; error bars are derived from the uncertainties in the magnitudes and the parallax. 


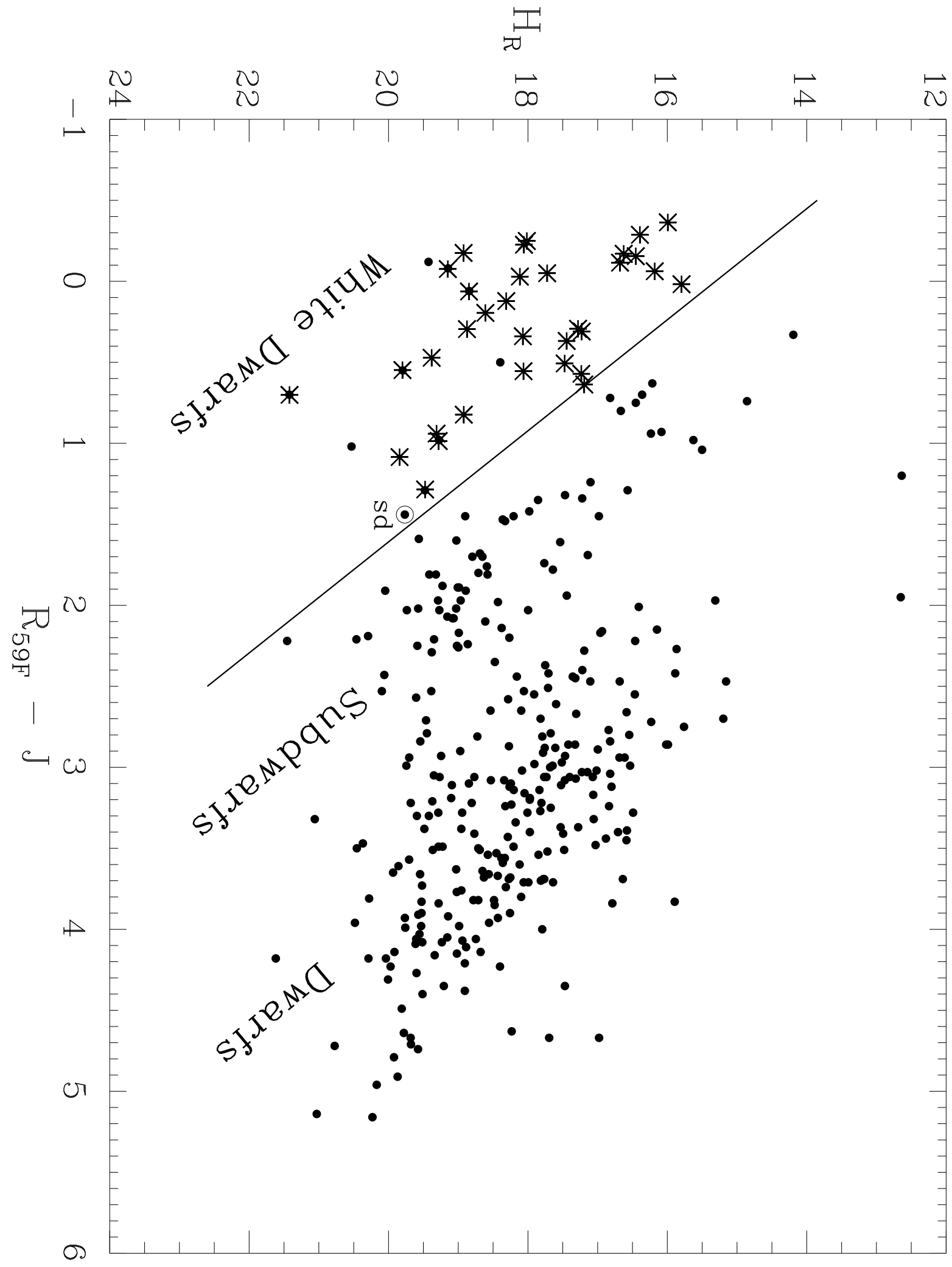




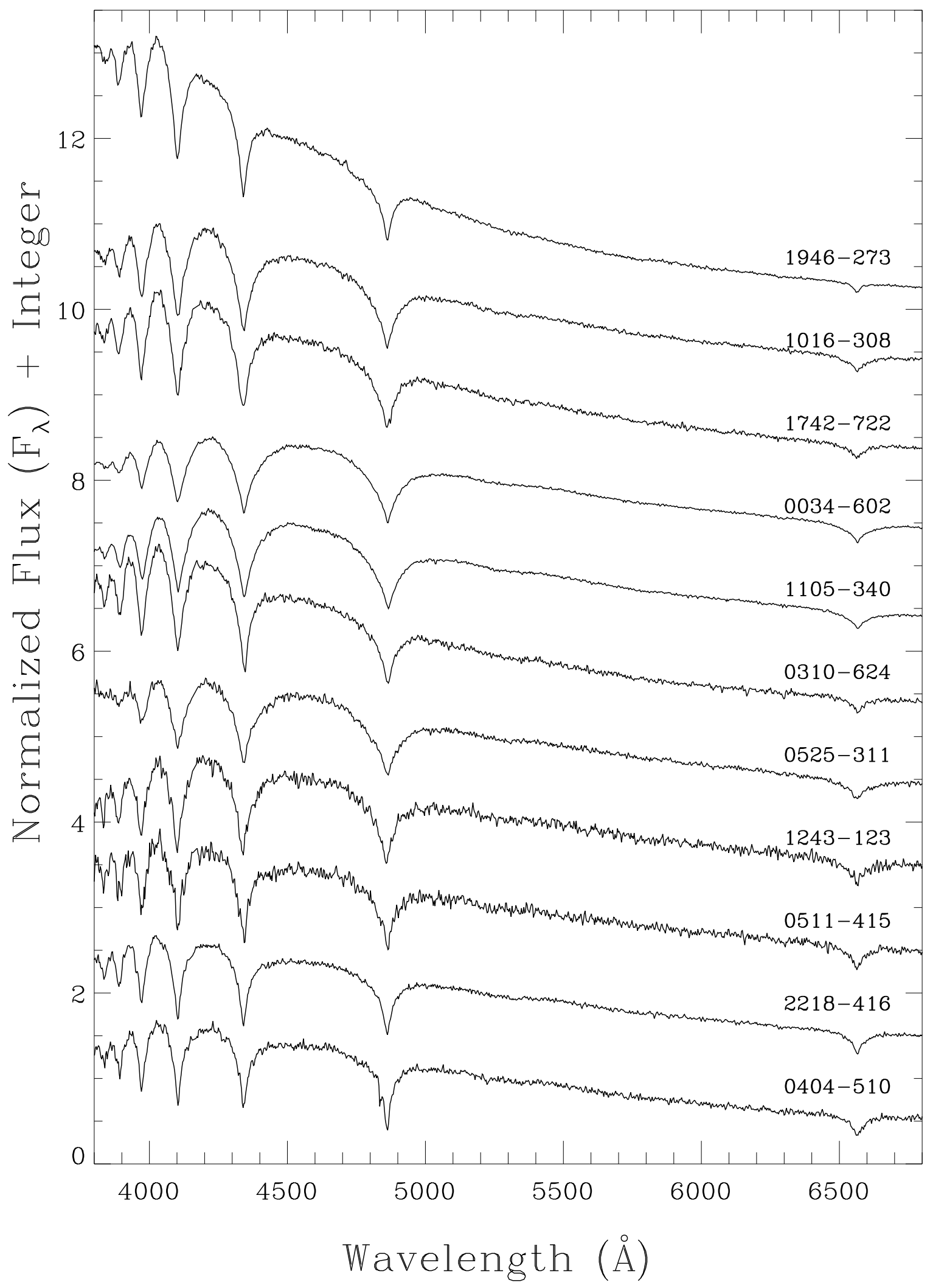




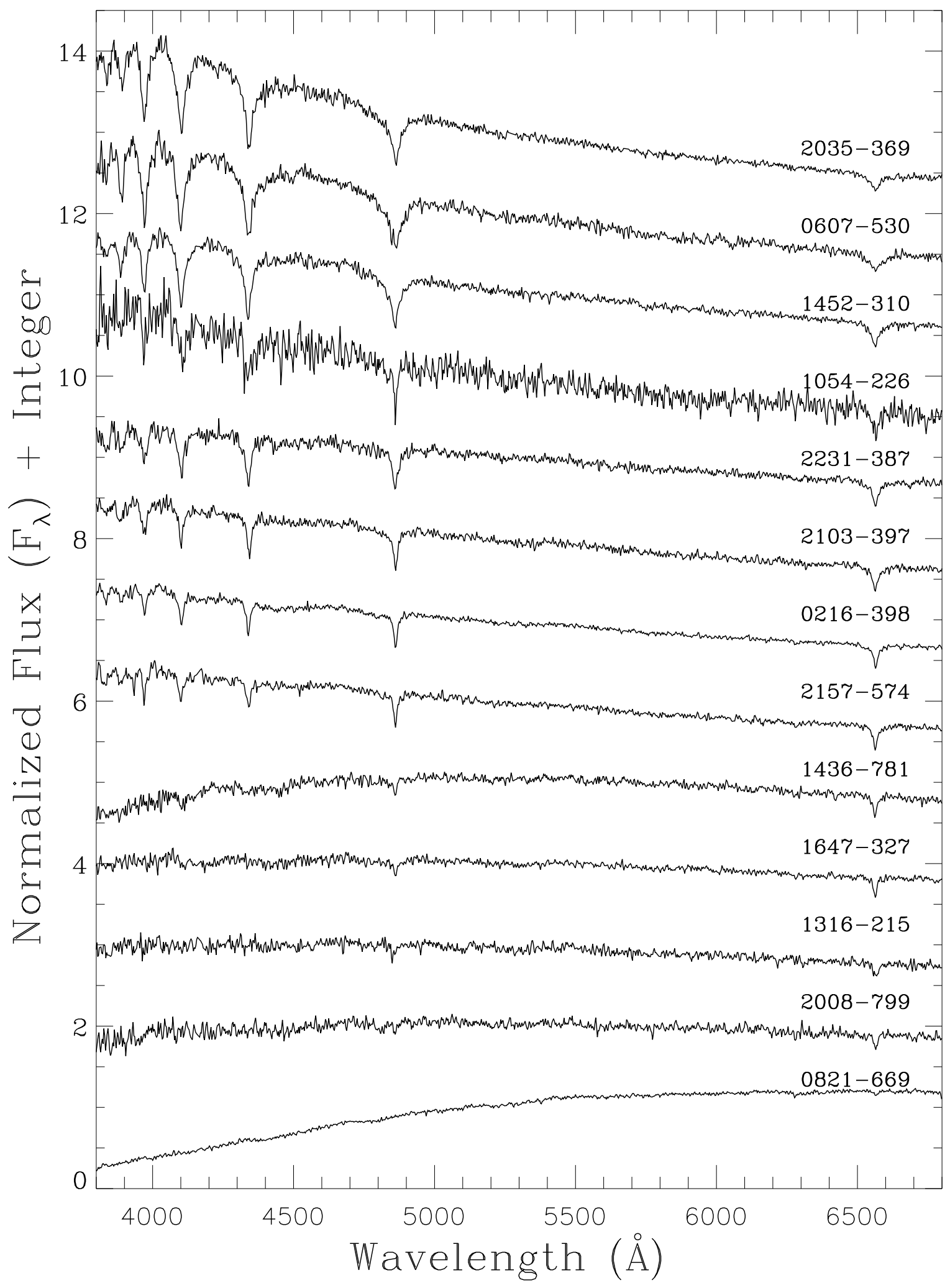




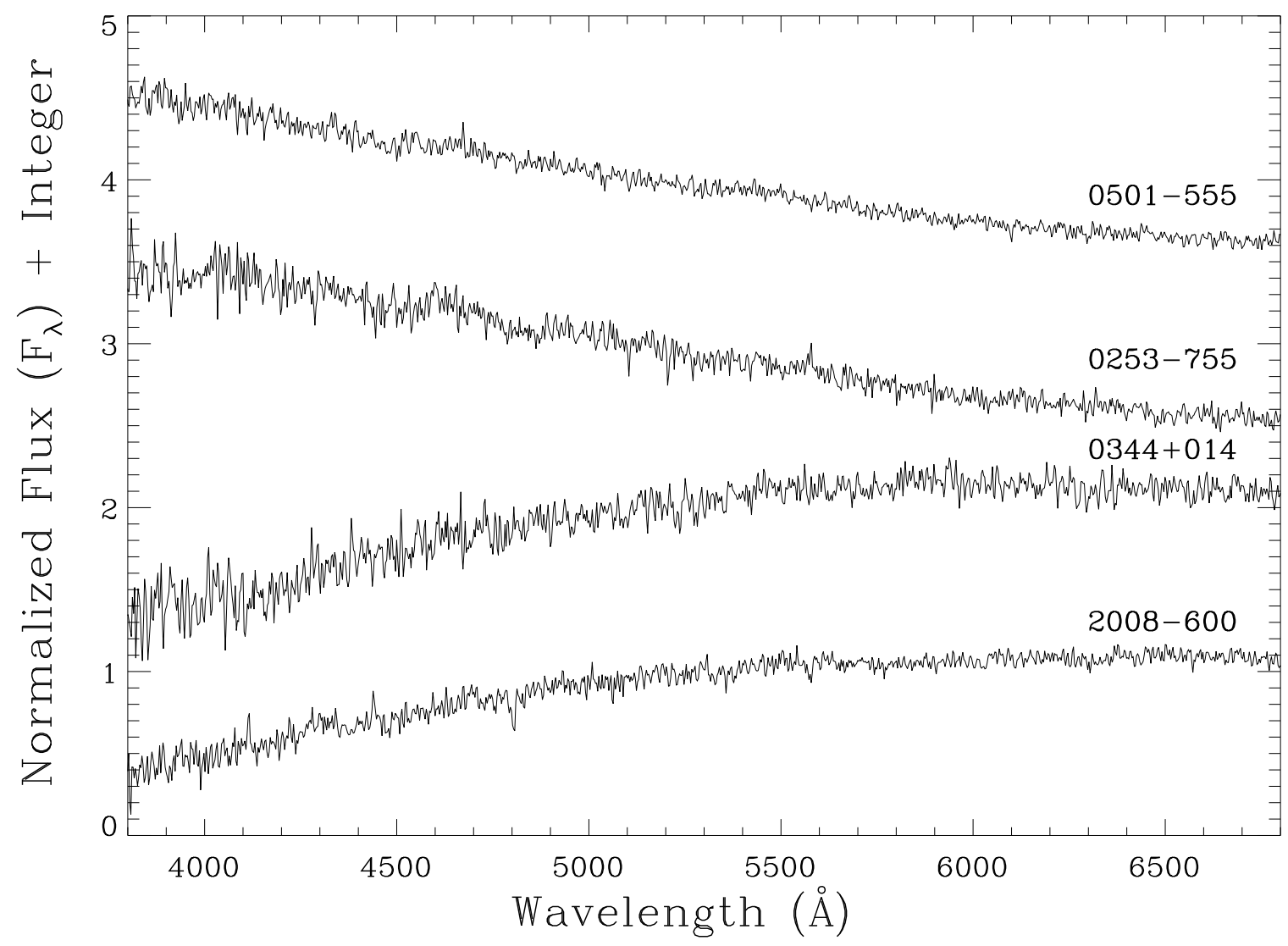




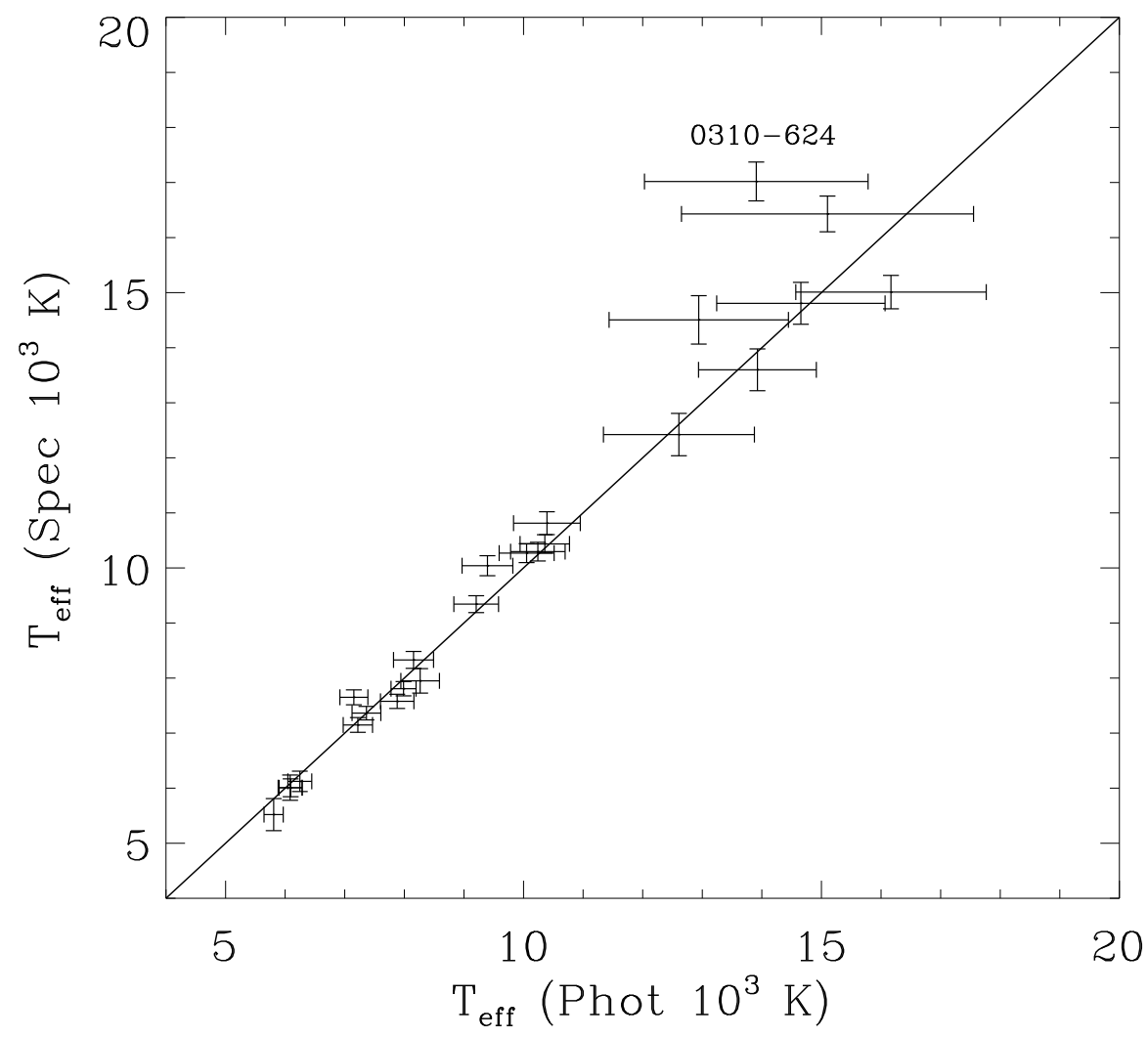




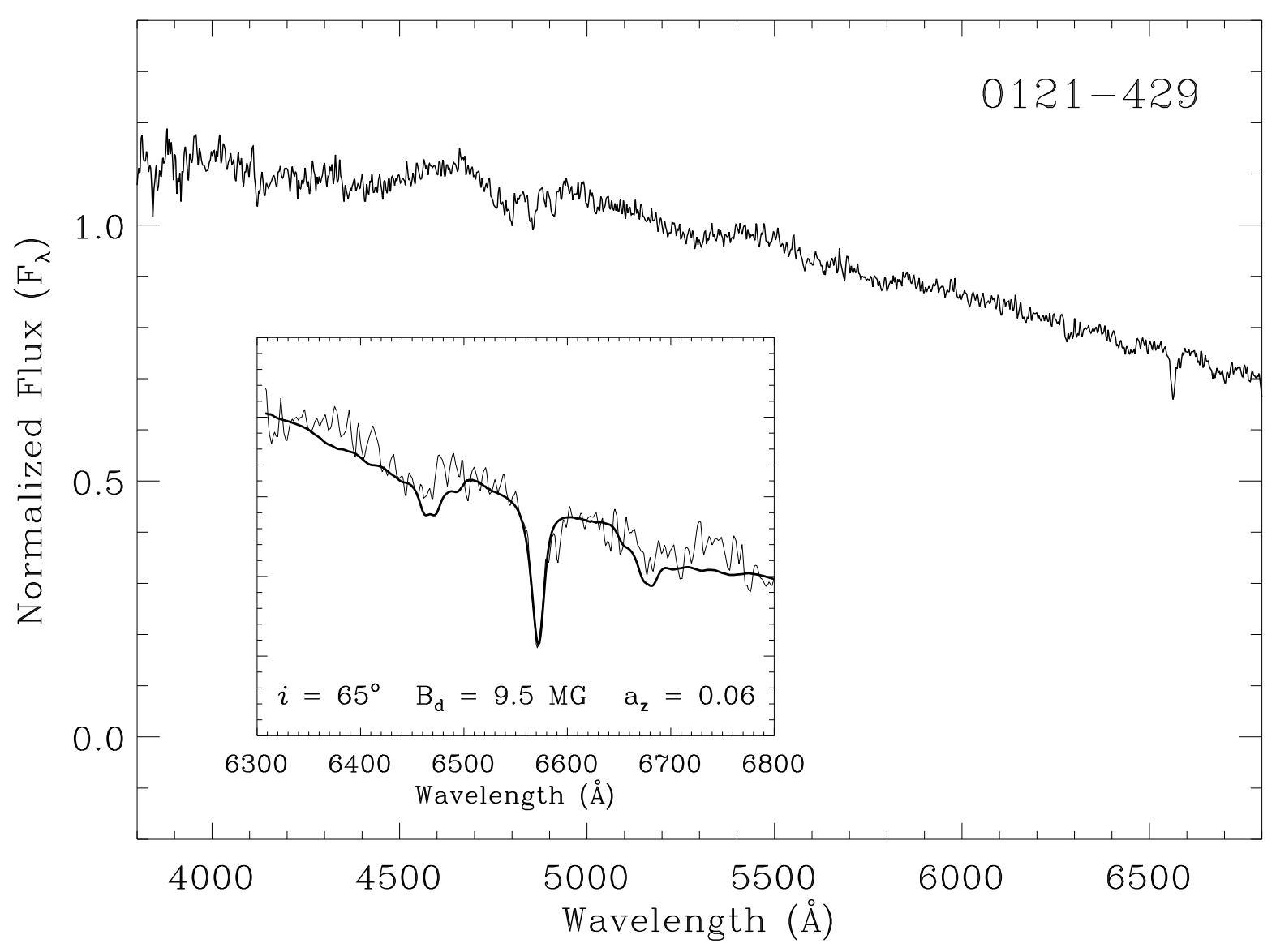




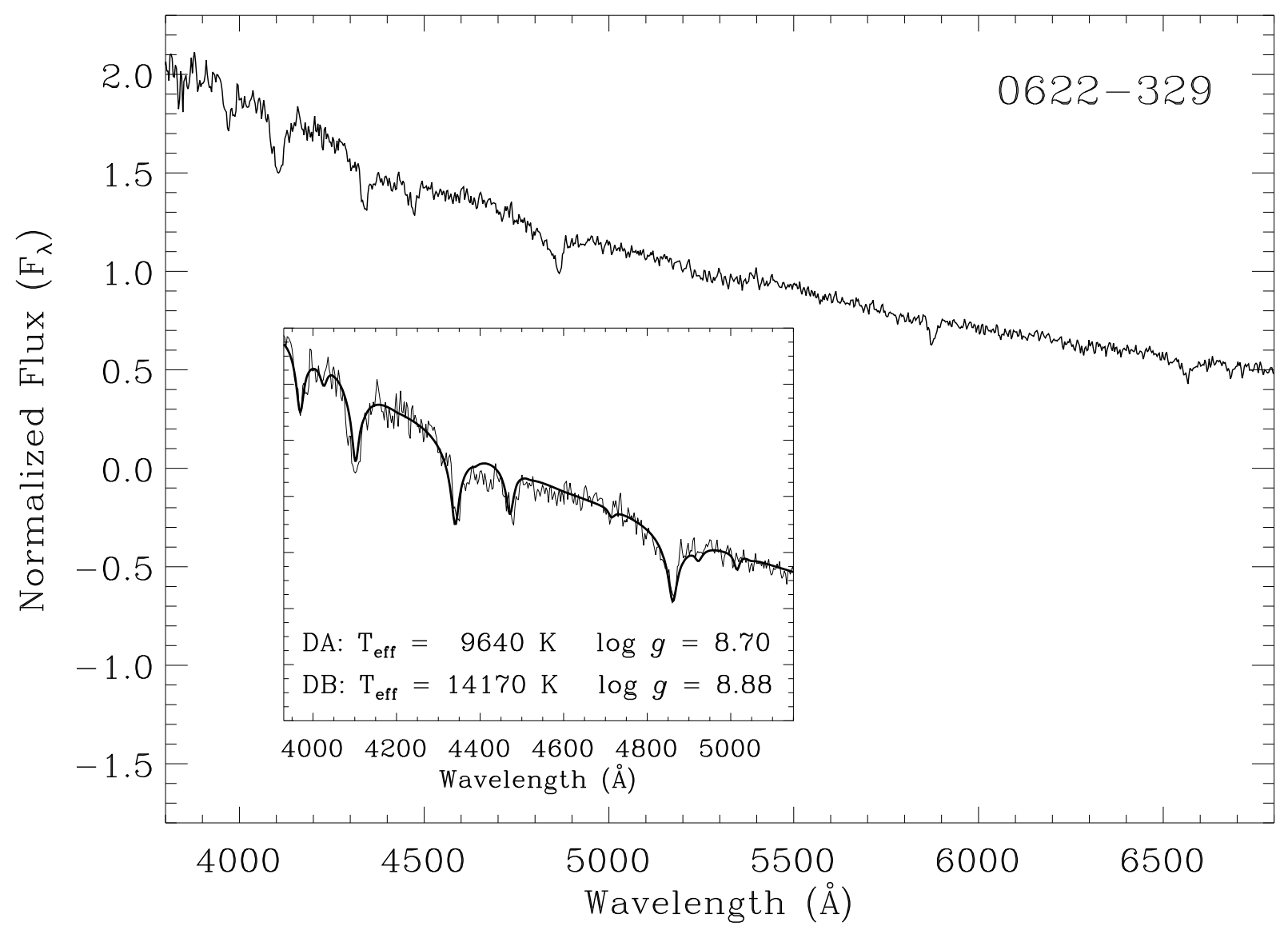




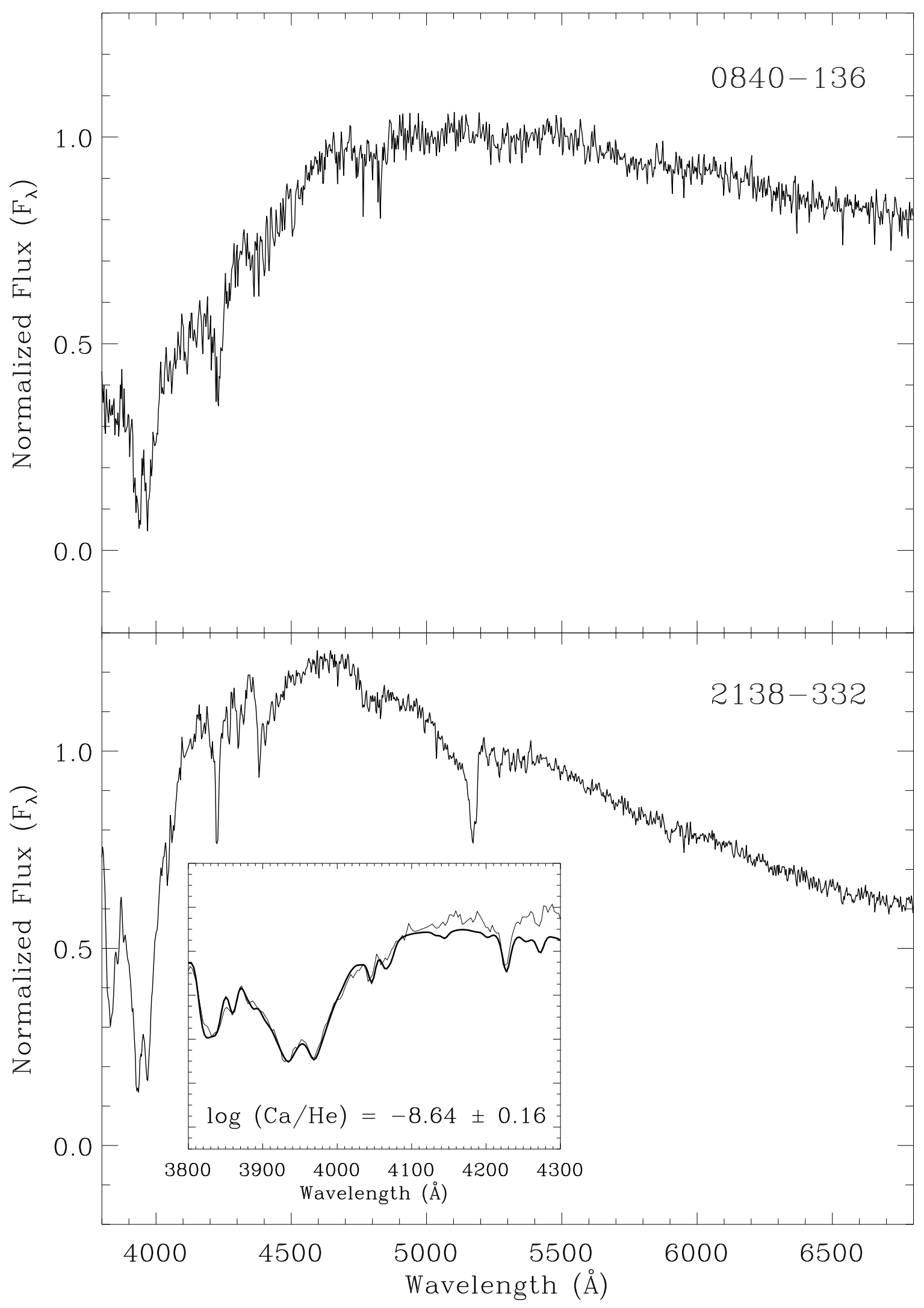




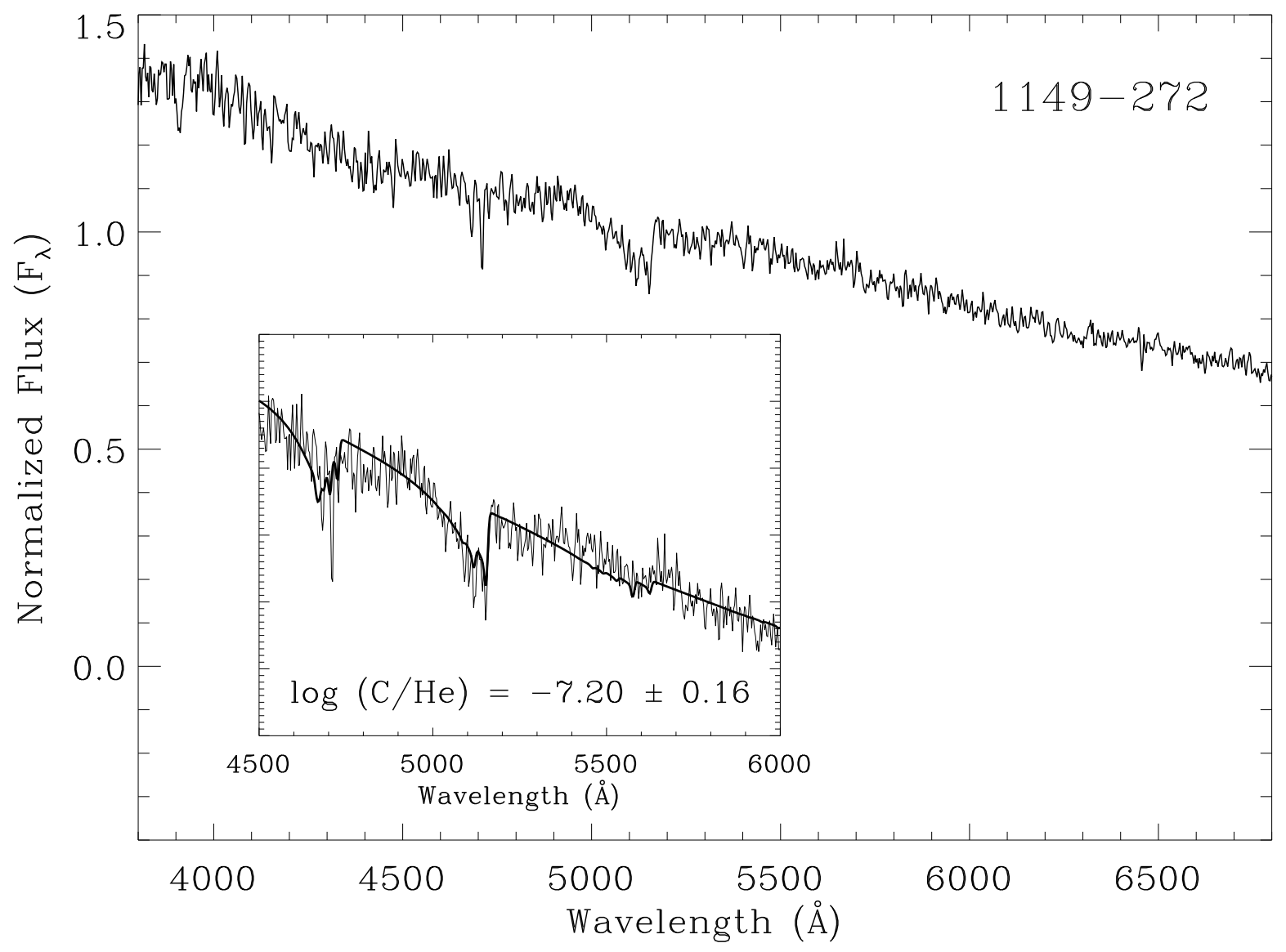




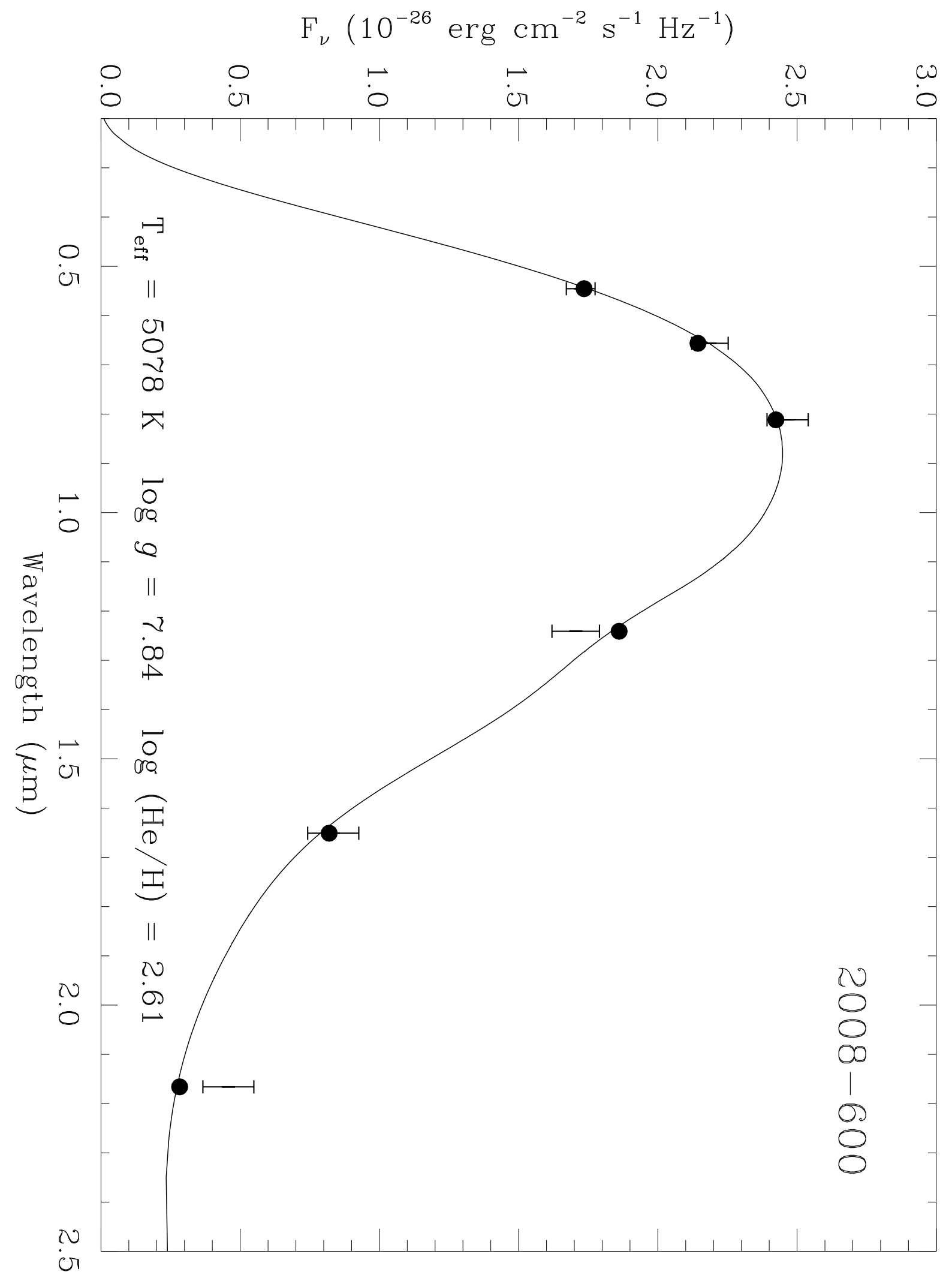

